\title{
A Nuclear Structure Model for Double Charge-Exchange Processes
}

\author{
V. dos S. Ferreira ${ }^{1}$, A. R. Samana ${ }^{2}$, F. Krmpotic ${ }^{3}$, and M. Chiapparini ${ }^{1}$ \\ ${ }^{1}$ Instituto de Física, Universidade do Estado do Rio de Janeiro, CEP 20550-900, Rio de Janeiro-RJ, Brazil \\ ${ }^{2}$ Departamento de Ciências Exactas e Tecnológicas, \\ Universidade Estadual de Santa Cruz, CEP 45662-000 Ilhéus, Bahia-BA, Brazil and \\ ${ }^{3}$ Instituto de Física La Plata, CONICET, Universidad Nacional de La Plata, 1900 La Plata, Argentina.
}

\begin{abstract}
A new model, based on the BCS approach, is specially designed to describe nuclear phenomena $(A, Z) \rightarrow(A, Z \pm 2)$ of double-charge exchange (DCE). After being proposed, and applied in the particle-hole limit, by one of the authors (F. Krmpotić [1]), so far it was never been applied within the BCS mean-field framework, nor has its ability to describe DCE processes been thoroughly explored. It is a natural extension of the pn-QRPA model, developed by Halbleib and Sorensen [2] to describe the single $\beta$-decays $(A, Z) \rightarrow(A, Z \pm 1)$, to the DCE processes. As such, it exhibits several advantages over the pn-QRPA model when is used in the evaluation of the double beta decay (DBD) rates. For instance, i) the extreme sensitivity of the nuclear matrix elements (NMEs) on the model parametrization does not occur, ii) it allows to study NMEs, not only for the fundamental state in daughter nuclei, as the pn-QRPA model does, but also for all final $0^{+}$and $2^{+}$states, accounting at the same time their excitation energies and the corresponding DBD Q-values, iii) together with the DBD-NMEs it provides also the energy spectra of Fermi and Gamow-Teller DCE transition strengths, as well as the locations of the corresponding resonances and their sum rules, iv) the latter are relevant for both the DBD and the DCE reactions, since the involved nuclear structure is the same; this correlation does not exist within the pn-QRPA model. As an example, detailed numerical calculations are presented for the $(A, Z) \rightarrow(A, Z+2)$ process in ${ }^{48} \mathrm{Ca} \rightarrow{ }^{48} \mathrm{Ti}$ and the $(A, Z) \rightarrow(A, Z-2)$ process in ${ }^{96} \mathrm{Ru} \rightarrow{ }^{96} \mathrm{Mo}$, involving all final $0^{+}$states and $2^{+}$states.
\end{abstract}

\section{INTRODUCTION}

The Double Charge-Exchange (DCE) processes relate the $(A, Z)$ nuclei with the $(A, Z+2)$ and $(A, Z-2)$ nuclei and will be labeled as $\{+2\}$ and $\{-2\}$ processes respectively.

The most studied DCE process is the Double Beta Decay (DBD). It is the slowest physical process observed so far, and can be used to learn about neutrino physics, provided we know how to deal with the nuclear structure. According with the number and type of leptons we may have the following DBD modes: i) double-electron decay $\left(2 \beta^{-}\right)$, ii) double-positron decay, iii) electron capturepositron emitting decay $\left(e \beta^{+}\right)$, and vi) double electron capture decay $(e e)$. Each of these decays occurs either with the emission of two neutrinos $(2 \nu$-decay) or they are neutrinoless $(0 \nu$-decay). To simplify the notation and when it does not cause confusion, we will designate the first process as $\mathrm{DBD}^{-}$and the remaining three as $\mathrm{DBD}^{+}$.

The $0 \nu$-decay rates depend on several unknown parameters such as neutrino mass, Majoron coupling, the coupling constants of the right-handed components of the weak hamiltonian, etc.) and the only way to put these in evidence is by having sufficient command over the nuclear structure. It is precisely at this point that the $2 \nu 2 \beta^{\mp}$, $2 \nu e \beta^{+}$, and $2 \nu e e$ decay modes are important. A comparison between experiment and theory for them provides a measure of the confidence that one may have in the nuclear wave functions employed for extracting the unknown parameters from $0 \nu$-lifetime measurements.

The number of possible candidates for $2 \beta^{-}$-decay is quite large: there are 35 nuclei. In addition, 34 nuclei can undergo $2 e$-electron capture, while 22 and 6 nuclei can undergo $e \beta^{+}$and $2 \beta^{+}$-decays respectively [3]. The discovery of the massiveness of the neutrino, through the observation of oscillations, boosted the importance of the $0 \nu$-DBD, since they are the only observables capable of providing the magnitude of the effective neutrino mass.

It is well known that the involved nuclear structure in $2 \nu$ and $0 \nu$-DBD is the same one that describes the DCE reactions. This fact reignited recently the interest in the measurements of heavy-ion-induced DCE reactions, such as the NUMEN project $[4,5]$ involving the ${ }^{40} \mathrm{Ca}\left({ }^{18} \mathrm{O},{ }^{18} \mathrm{Ne}\right){ }^{40} \mathrm{Ar}$ process. Simultaneously, the interest in the theoretical study of the DCE reactions has been renewed [6, 7]. More, Shimizu, Menéndez, and Yako [8] have also latterly noted correlations between the DCE and $0 \nu 2 \beta^{-}$-decay. In all the mentioned theoretical studies, the calculations were made within the framework of the shell model (SM).

The neutrinoless DBDs occur in medium-mass nuclei that are often far from closed shells and, as a consequence, the calculations are mostly made in the proton-neutron Quasiparticle Random Phase Approximation (pn-QRPA), since this tool is computationally much more simple than the SM. As discussed in Ref. [9], the kind of correlations that these two methods include are not the same. The pn-QRPA deals with a large fraction of nucleons in a large single-particle space, but within a modest configuration space. The SM, by contrast, deals with a small fraction of nucleons in a limited single-particle space, but allows them to correlate in arbitrary ways within a large configuration space. There is another important difference. The standard pn-QRPA only allows us to calculate the double-charge exchange transitions from the ground state of the decaying $(A, Z)$ 


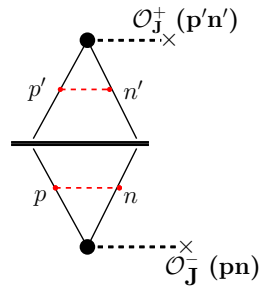

(a)
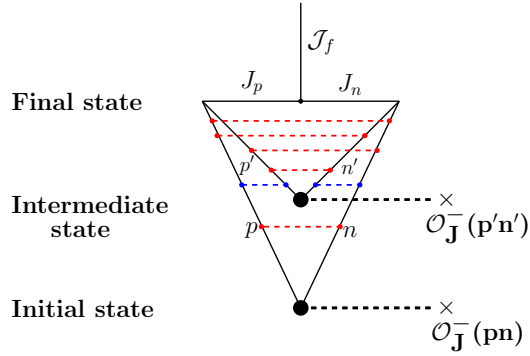

(b)
FIG. 1: (Color online) Graphical representation of the numerators in the $2 \beta^{-}$NME, where the black points indicate the single $\beta$-decays. They are: a) $\left\langle 0_{f}^{+}\left\|\mathcal{O}_{J}^{+}\right\| J_{\alpha}^{+}\right\rangle\left\langle J_{\alpha}^{+} \mid J_{\alpha^{\prime}}^{+}\right\rangle\left\langle J_{\alpha^{\prime}}^{+} \| \mathcal{O}_{J}^{-} \mid 0_{i}^{+}\right\rangle$in the pn-QRPA model, where the overlap between the initial and final QRPA solutions for the intermediate nucleus, $\left\langle J_{\alpha}^{+} \mid J_{\alpha^{\prime}}^{+}\right\rangle$, is represented by a thick line, and b) $\left\langle\mathcal{J}_{f}^{+} \| \mathcal{O}_{J}^{-}|| J_{\alpha}^{+}\right\rangle\left\langle J_{\alpha}^{+} \| \mathcal{O}_{J}^{-} \mid 0_{i}^{+}\right\rangle$in the (pn,2p2n)-QTDA model, which appears in Eqs. (2.9) and (2.15), and indicates that the first $\beta^{-}$-decay is switched on in the initial state and the second in the intermediate state. The $2 \beta^{+}$NMEs are represented in the same way after making the substitution $\mathcal{O}_{J}^{\mp} \leftrightarrow \mathcal{O}_{J}^{ \pm}$. The pn, and nn + pp nuclear interactions between protons and neutrons are indicated by red and blue dashed lines, respectively. The five vertices of the diagram b) correspond to five of six angular momentum coupling in the symbol 9j in Eq. (2.47). The sixth coupling $(J J) \mathcal{J}$ corresponds to the three unconnected lines in this figure.

nucleus to the ground state in the final $(A, Z \pm 2) \mathrm{nu}-$ clei. In fact, to evaluate the transitions going to the excited states, a second ( $p p+n n)$-QRPA must to be performed [10, 11], which introduces additional free parameters and it is limited to one and two quadrupole phonon states.

To deal with the DCE processes we will resort here to a Tamm-Dancoff Approximation (TDA), which has been suggested and discussed on its $p h$-limit for ${ }^{48} \mathrm{Ca}$ more than a decade ago in Ref. [1]. In this model is assumed that the initial, intermediate and final nuclei are the BCS vacuum, pn-excitations, and 2p2n-excitations respectively. The resulting model will be labeled as (pn,2p2n)-QTDA. The main differences with the standard pn-QRPA model are illustrated in Fig. 1.

The present model is a natural extension to double charge-exchange processes of the pn-QRPA model, originally proposed by Halbleib and Sorensen (HS) in 1967 to describe the single $\beta$-decays [2]. As such, it allows to evaluate the NME, not only for the ground states but also of all final $0^{+}$and $2^{+}$states, as well as the $Q$-values for the $2 \beta^{-}$-decay $\left(Q_{2 \beta^{-}}\right)$, and for the $2 e$-capture $\left(Q_{2 e}\right)$. It yields as well the DCE energy strength spectra and their sum rules, which are relevant for associated reaction processes and resonances. Detailed numerical calculations are performed in the present work for the ${ }^{48} \mathrm{Ca} \rightarrow{ }^{48} \mathrm{Ti}$, and ${ }^{96} \mathrm{Ru} \rightarrow{ }^{96}$ Mo processes, involving their final $0^{+}$and $2^{+}$states.

\section{FORMALISM}

\section{A. Nuclear matrix elements and double charge-exchange excitations}

Independently of the nuclear model used, and when are only considered the allowed transitions, i.e. the GamowTeller (GT) and Fermi (F) transitions, the NMEs for the $2 \nu 2 \beta^{ \pm}$-decay, from the ground state $0_{i}^{+}$in the initial nucleus $(A, Z)$ to one of the states $0_{f}^{+}$in the final nuclei $(A, Z \mp 2)$, reads ${ }^{1}$

$$
\begin{gathered}
M^{2 \nu^{ \pm}}\left(0_{f}^{+}\right)=M_{F}^{2 \nu^{ \pm}}\left(0_{f}^{+}\right)+M_{G T}^{2 \nu^{ \pm}}\left(0_{f}^{+}\right) \\
\equiv \sum_{J=0,1}(-)^{J} g_{J}^{2} \sum_{\alpha}\left[\frac{\left\langle 0_{f}^{+}\left\|\mathcal{O}_{J}^{ \pm}\right\| J_{\alpha}^{+}\right\rangle\left\langle J_{\alpha}^{+}\left\|\mathcal{O}_{J}^{ \pm}\right\| 0_{i}^{+}\right\rangle}{\mathcal{D}_{J_{\alpha}, 0_{f}}^{2 \nu^{ \pm}}}\right]
\end{gathered}
$$

where $g_{0} \equiv g_{V}$ and $g_{1} \equiv g_{A}$ are the vector and axial-vector weak coupling constants respectively, and the summation goes over all intermediate virtual states $\left|J_{\alpha}^{+}\right\rangle$in the nuclei $(A, Z \mp 1)$.

The one-body operators are

$$
\begin{aligned}
& \mathcal{O}_{J}^{-}=\hat{J}^{-1} \sum_{p n}\left\langle p\left\|\mathrm{O}_{J}\right\| n\right\rangle\left(c_{p}^{\dagger} c_{\bar{n}}\right)_{J}, \\
& \mathcal{O}_{J}^{+}=\hat{J}^{-1} \sum_{p n}\left\langle n\left\|\mathrm{O}_{J}\right\| p\right\rangle\left(c_{n}^{\dagger} c_{\bar{p}}\right)_{J},
\end{aligned}
$$

with $\mathrm{O}_{0}=1$, and $\mathrm{O}_{1}=\sigma$ for $\mathrm{F}$ and GT transitions, respectively, and $c_{k}^{\dagger} \equiv c_{j_{k}, m_{k}}^{\dagger}$ and $c_{\bar{k}} \equiv(-)^{j_{k}-m_{k}} c_{j_{k},-m_{k}}$ being the single-particle creation and annihilation operators, and $\hat{J}=\sqrt{2 J+1}$.

The energy denominator in (2.1) is ${ }^{2}$

$$
\begin{aligned}
\mathcal{D}_{J_{\alpha}, \mathcal{J}_{f}}^{2 \nu^{ \pm}} & =E_{J_{\alpha}}^{\{\mp 1\}}-\frac{E_{0^{+}}^{\{0\}}+E_{\mathcal{J}_{f}}^{\{\mp 2\}}}{2} \\
& =E_{J_{\alpha}}^{\{\mp 1\}}-E_{0^{+}}^{\{0\}}+\frac{E_{0^{+}}^{\{0\}}-E_{\mathcal{J}_{f}}^{\{\mp 2\}}}{2}
\end{aligned}
$$

where $E_{0^{+}}^{\{0\}}, E_{J_{\alpha}}^{\{ \pm 1\}}$ and $E_{\mathcal{J}_{f}}^{\{ \pm 2\}}$ are the energies of the decaying $(A, Z)$ nucleus in its ground state, the interme-

\footnotetext{
${ }^{1}$ For the first-forbidden NME see Ref. [12].

2 The last term in the denominator (2.3) is based on the assumption that the lepton energies can be replaced by $e+\nu \cong$ $\left(E_{0^{+}}^{\{0\}}-E_{\mathcal{J}_{f}}^{\{\mp 2\}}\right) / 2$, whose validity for the mixed mode was questioned by Hirsch et. al [50]. Following their idea on equal sharing of the liberated energy among the emitted leptons, Suhonen [10] has derived formulas for the three different $\mathrm{DBD}^{+}$, and has used them in the evaluation of the decay ${ }^{96} \mathrm{Ru} \rightarrow{ }^{96} \mathrm{Mo}$. Unfortunately, the author has omitted a factor of 2 in his Eq.(13), which makes wrong the numerical result of mode $2 \beta^{+}$. The same error was propagated in subsequent works $[14,15]$. Here we will continue using the estimate (2.3).
} 
diate $(A, Z \pm 1)$ nuclei in the state $J_{\alpha}^{+}$, and final $(A, Z \pm 2)$ nuclei in the state $\mathcal{J}_{f}^{+}$, respectively.

The matrix elements $\left\langle J_{\alpha}^{+}\left\|\mathcal{O}_{J}^{ \pm}\right\| 0_{i}^{+}\right\rangle$can be expressed as a product of model dependent one-body densities

$$
\begin{aligned}
& \rho^{-}\left(p n J_{\alpha}\right)=\hat{J}^{-1}\left\langle J_{\alpha}\left\|\left(c_{p}^{\dagger} c_{\bar{n}}\right)_{J}\right\| 0_{i}^{+}\right\rangle, \\
& \rho^{+}\left(p n J_{\alpha}\right)=\hat{J}^{-1}\left\langle J_{\alpha}\left\|\left(c_{n}^{\dagger} c_{\bar{p}}\right)_{J}\right\| 0_{i}^{+}\right\rangle,
\end{aligned}
$$

and a purely geometric (angular) factors ${ }^{3}$

$$
\begin{aligned}
& O_{J}(p n) \equiv\left\langle p\left\|\mathrm{O}_{J}\right\| n\right\rangle \\
= & \delta_{l_{p} l_{n}} \sqrt{2} \hat{J} \hat{j}_{n} \hat{j}_{p}(-)^{j_{p}+l_{p}+J+\frac{1}{2}}\left\{\begin{array}{ccc}
j_{p} & j_{n} & J \\
\frac{1}{2} & \frac{1}{2} & l_{n}
\end{array}\right\},
\end{aligned}
$$

which is the single-particle NME, with $O_{J}(n p)=$ $(-)^{j_{n}-j_{p}} O_{J}(p n)$. Namely,

$$
\left\langle J_{\alpha}^{+}\left\|\mathcal{O}_{J}^{ \pm}\right\| 0_{i}^{+}\right\rangle=\sum_{p n} \rho^{ \pm}\left(p n J_{\alpha}^{\pi}\right) O_{J}(p n) .
$$

Similarly,

$$
\left\langle\mathcal{J}_{f}^{+}\left\|\mathcal{O}_{J}^{ \pm}\right\| J_{\alpha}^{+}\right\rangle=\sum_{n p} \rho^{ \pm}\left(p n J_{\alpha}, \mathcal{J}_{f}^{+}\right) O_{J}(p n),
$$

where

$$
\begin{aligned}
& \rho^{+}\left(p_{n} J_{\alpha}, \mathcal{J}_{f}^{+}\right)=\hat{J}^{-1}\left\langle\mathcal{J}_{f}^{+}\left\|\left(c_{n}^{\dagger} c_{\bar{p}}\right)_{J}\right\| J_{\alpha}\right\rangle, \\
& \rho^{-}\left(p n J_{\alpha}, \mathcal{J}_{f}^{+}\right)=\hat{J}^{-1}\left\langle\mathcal{J}_{f}^{+}\left\|\left(c_{p}^{\dagger} c_{\bar{n}}\right)_{J}\right\| J_{\alpha}\right\rangle,
\end{aligned}
$$

are the corresponding density matrices for transitions from the intermediate states $\left|J_{\alpha}\right\rangle$ to the final states $\left|\mathcal{J}_{f}^{+}\right\rangle$, with $\mathcal{J}=0,2$.

The corresponding matrix elements are:

$$
\begin{aligned}
& M_{F}^{2 \nu^{ \pm}}\left(0_{f}^{+}\right)=g_{V}^{2} \sum_{\alpha} \frac{\left\langle 0_{f}^{+} \| \mathcal{O}_{0}^{ \pm}|| 0_{\alpha}^{+}\right\rangle\left\langle 0_{\alpha}^{+} \| \mathcal{O}_{0}^{ \pm} \mid 0_{i}^{+}\right\rangle}{\mathcal{D}_{0_{\alpha}^{+}, 0_{f}^{+}}^{2 \nu^{ \pm}}}, \\
& M_{G T}^{2 \nu^{ \pm}}\left(\mathcal{J}_{f}^{+}\right)=\frac{-g_{A}^{2}}{\sqrt{\mathcal{J}+1}} \sum_{\alpha} \frac{\left\langle\mathcal{J}_{f}^{+}\left\|\mathcal{O}_{1}^{ \pm}\right\| 1_{\alpha}^{+}\right\rangle\left\langle 1_{\alpha}^{+} \| \mathcal{O}_{1}^{ \pm} \mid 0_{i}^{+}\right\rangle}{\left(\mathcal{D}_{1_{\alpha}^{+}, \mathcal{J}_{f}^{+}}^{2 \nu^{ \pm}}\right)^{\mathcal{J}+1}}
\end{aligned}
$$

where the GT-NMEs to $2_{f}^{+}$states have also been included [16-18].

All the information about the nuclear structure is contained in the one-body density matrices (2.4) and (2.8), or more precisely in the two-body density matrices

$$
\rho^{ \pm}\left(p n p^{\prime} n^{\prime} ; J_{\alpha}, \mathcal{J}_{f}^{+}\right)=\rho^{ \pm}\left(p n ; J_{\alpha}\right) \rho^{ \pm}\left(p^{\prime} n^{\prime} ; J_{\alpha}, \mathcal{J}_{f}^{+}\right) .
$$

\footnotetext{
${ }^{3}$ We use here the angular momentum coupling scheme $\left|\left(\frac{1}{2}, l\right) j\right\rangle$.
}

The NME for the $0 \nu 2 \beta^{ \pm}$-decays to the $0_{f}^{+}$final states can be easily evaluated from these densities. In fact, after doing in [19, Eqs. (2.20)] the replacement

$$
\rho^{p h}\left(p n p^{\prime} n^{\prime} ; J_{\alpha}\right) \rightarrow \rho^{ \pm}\left(p n p^{\prime} n^{\prime} ; J_{\alpha}, 0_{f}^{+}\right),
$$

we can express them as

$$
M^{0 \nu^{ \pm}}\left(0_{f}^{+}\right)=\sum_{X} M_{X}^{0 \nu^{ \pm}}\left(0_{f}^{+}\right)
$$

where $X=V, A, P, M$ stands for vector $(V)$, axial-vector $(A)$, pseudo-scalar $(P)$, and weak-magnetism $(M)$ terms. We proceed in the same way with the NME $M^{0 \nu^{ \pm}}\left(2_{f}^{+}\right)$.

It is well known that the single $\beta$-decay processes to the states $J_{\alpha}$ in $(A, Z+1)$ and $(A, Z-1)$ nuclei are related to the following single charge-exchange transition strengths

$$
S_{J}^{\{ \pm 1\}} \equiv \sum_{\alpha} B_{J_{\alpha}}^{\{ \pm 1\}}=\hat{J}^{-2} \sum_{\alpha}\left|\left\langle J_{\alpha}^{+}\left\|\mathcal{O}_{J}^{\mp}\right\| 0_{i}^{+}\right\rangle\right|^{2} .
$$

When $\left|J_{\alpha}^{+}\right\rangle$is a complete set of excited states that can be reached by operating with $\mathcal{O}_{J}^{ \pm}$on the initial state $\left|0_{i}^{+}\right\rangle$, they satisfy the single-charge exchange (SCE) sume rule or Ikeda sum rule, for both the F and GT transitions,

$$
\begin{aligned}
& S_{J}^{\{1\}} \equiv S_{J}^{\{+1\}}-S_{J}^{\{-1\}} \\
= & (-)^{J} \hat{J}^{-2}\left\langle 0_{i}^{+}\left|\left[\mathcal{O}_{J}^{+}, \mathcal{O}_{J}^{-}\right]_{0}\right| 0_{i}^{+}\right\rangle=N-Z .
\end{aligned}
$$

Similarly, both $M^{2 \nu 2 \beta^{ \pm}}\left(\mathcal{J}_{f}^{+}\right)$and $M^{0 \nu 2 \beta^{ \pm}}\left(\mathcal{J}_{f}^{+}\right)$are related to the double-charge-exchange operators $\left(\mathcal{O}_{J}^{ \pm} \mathcal{O}_{J}^{ \pm}\right)_{\mathcal{J}}$ and to their spectral distributions in nuclei $(A, Z \pm 2)$ nuclei given by

$$
\begin{aligned}
S_{J \mathcal{J}}^{\{ \pm 2\}} & \equiv \sum_{f} B_{J \mathcal{J}_{f}}^{\{ \pm 2\}} \\
& =\hat{J}^{-2} \sum_{f}\left|\sum_{\alpha}\left\langle\mathcal{J}_{f}^{+}\left\|\mathcal{O}_{J}^{\mp}\right\| J_{\alpha}^{+}\right\rangle\left\langle J_{\alpha}^{+} \| \mathcal{O}_{J}^{\mp} \mid 0_{i}^{+}\right\rangle\right|^{2} .
\end{aligned}
$$

When both $\left|J_{\alpha}^{+}\right\rangle$and $\left|\mathcal{J}_{f}^{+}\right\rangle$are complete set of excited states that can be reached by operating with $\mathcal{O}_{J}^{ \pm}$, and $\left(\mathcal{O}_{J}^{ \pm} \mathcal{O}_{J}^{ \pm}\right)_{\mathcal{J}}$ on the initial state $\left|0_{i}^{+}\right\rangle$, their differences

$$
\begin{aligned}
S_{J \mathcal{J}}^{\{2\}} & =S_{J \mathcal{J}}^{\{+2\}}-S_{J \mathcal{J}}^{\{-2\}} \\
& =\hat{J}^{-2} \sum_{f}\left[\left|\sum_{\alpha}\left\langle\mathcal{J}_{f}^{+}\left\|\mathcal{O}_{J}^{-}\right\| J_{\alpha}^{+}\right\rangle\left\langle J_{\alpha}^{+} \| \mathcal{O}_{J}^{-} \mid 0_{i}^{+}\right\rangle\right|^{2}\right. \\
& \left.-\left|\sum_{\alpha}\left\langle\mathcal{J}_{f}^{+}\left\|\mathcal{O}_{J}^{+}\right\| J_{\alpha}^{+}\right\rangle\left\langle J_{\alpha}^{+} \| \mathcal{O}_{J}^{+} \mid 0_{i}^{+}\right\rangle\right|^{2}\right]
\end{aligned}
$$

obey the double-charge-exchange sum rules (DCESR), which were evaluated in Refs. $[6,7,20-22]$ with the following results:

$$
\mathrm{S}_{D F} \equiv \mathrm{S}_{00}^{\{2\}}=2(N-Z)(N-Z-1),
$$




$$
\begin{aligned}
\mathrm{S}_{D G T, 0} & \equiv \mathrm{S}_{10}^{\{2\}} \\
& =2(N-Z)\left(N-Z+1+2 S_{1}^{\{+1\}}\right)-\frac{2}{3} C, \\
\mathrm{~S}_{D G T, 2} & \equiv \mathrm{S}_{12}^{\{2\}} \\
& =10(N-Z)\left(N-Z-2+2 S_{1}^{\{+1\}}\right)+\frac{5}{3} C,
\end{aligned}
$$

where $C$ is a relatively small quantity given by $[21, \mathrm{Eq}$. (4)]. These equations agree with the Eq. (8) in Ref. [7] except for a factor of 3 and the omission of $S_{1}^{\{+1\}}$.

Combining Eqs. (2.18) and (2.19) one obtains the sum rule for the total GT strength

$$
\mathrm{S}_{D G T}=12(N-Z)\left(N-Z-\frac{3}{2}+2 S_{1}^{\{+1\}}\right)+C,
$$

which is independent of the structure of the ground-state wave function [21].

The relationship between the $\beta \beta$-decay and double charge exchange reactions has been discussed recently in Refs. [4, 8].

\section{B. (pn,2p2n)-QTDA Model}

The pn-QRPA evaluations of the $\beta \beta$-decays are generally limited to the ground state of the final nuclei, i.e. to the calculation of $M_{2 \nu}\left(0_{1}^{+}\right)$and $M_{0 \nu}\left(0_{1}^{+}\right)$. Moreover, the SCE sume rules (2.14) are fulfilled within this model, but it does not allow us to evaluate the strengths $S_{J \mathcal{J}}^{\beta \beta^{ \pm}}$ given by (2.16) and to discuss the corresponding DCESR listed in Eqs. (2.17)-(2.20).

To describe the intermediate states $J_{\alpha}^{+}$in both pnQRPA and (pn,2p2n)-QTDA models it is used a nuclear Hamiltonian of the type

$$
H_{1}=H_{0}+H_{p n}
$$

where

$$
H_{0}=\sum_{\alpha} E_{k} a_{k}^{\dagger} a_{k}
$$

is the independent-quasiparticle Hamiltonian, with $a_{\alpha}^{\dagger}$ and $a_{\bar{\alpha}}$ being the single-quasiparticle creation and annihilation operators, defined by the Bogoljubov transformation [23, Eqs. (13.10)]

$$
\begin{aligned}
& a_{k}^{\dagger}=u_{a} c_{k}^{\dagger}+v_{k} c_{\bar{k}}, \\
& a_{\bar{k}}=u_{a} c_{\bar{k}}-v_{a} c_{k}^{\dagger} .
\end{aligned}
$$

The proton and neutron pairing interactions are contained in the transformation coefficients $u_{a}$ and $v_{a}$, and in the quasiparticle energy

$$
E_{k}=\left[\left(e_{k}-\lambda\right)^{2}+\Delta_{k}^{2}\right]^{\frac{1}{2}}
$$

where $e_{k}$ is the shell-model single-particle energy (spe), and $\lambda$ is the chemical potential or Fermi level. The energy gap parameters $\Delta_{k}$ and the pairing coupling constants are determined to reproduce the experimental oddmass difference for each nucleus. Finally, $H_{p n}$ is the quasiproton-quasineutron interaction.

Within the pn-QRPA, the states $\left|J_{\alpha}\right\rangle$ with excitation energy $\omega_{J_{\alpha}}$, are created from the correlated initial and final $0^{+}$ground states by proton-neutron phonon creation operators $Q_{J_{\alpha}}$, which are defined as a linear superposition of creation and annihilation proton-neutron quasiparticle pair operators

$$
A^{\dagger}(p n J)=\left[a_{p}^{\dagger} a_{n}^{\dagger}\right]_{J}
$$

that is

$$
\begin{aligned}
\left|J_{\alpha}\right\rangle & =Q_{J_{\alpha}}^{\dagger}\left|0^{+}\right\rangle \\
& \equiv \sum_{p n}\left[X_{p n J}^{\alpha} A^{\dagger}(p n J)-Y_{p n J}^{\alpha} A(p n J)\right]\left|0^{+}\right\rangle
\end{aligned}
$$

and

$$
Q_{J_{\alpha}}\left|0^{+}\right\rangle=0, \quad H_{1}\left|J_{\alpha}\right\rangle=\omega_{J_{\alpha}}\left|J_{\alpha}\right\rangle
$$

Usually this is done for initial $\left|0_{I}^{+}\right\rangle$and final $\left|0_{F}^{+}\right\rangle$ ground states, obtaining two sets of intermediate states $J_{\alpha}$ and $J_{\alpha^{\prime}}$ in the $(N-1, Z+1)$ nucleus, which are different from each other. Therefore, in the evaluation of the $\beta \beta$-NME it is necessary to consider their overlap, which is indicated in Fig. 1, which corresponds to the substitution

$$
\begin{aligned}
\sum_{J_{\alpha}} & \rho^{p h}\left(p n p^{\prime} n^{\prime} ; J_{\alpha}\right) \rightarrow\left\langle 0_{I}^{+} \mid 0_{F}^{+}\right\rangle \times \\
& \sum_{J^{\pi} \alpha \alpha^{\prime}} \rho^{+}\left(p^{\prime} n^{\prime} ; J_{\alpha^{\prime}}\right)\left\langle J_{\alpha^{\prime}} \mid J_{\alpha}\right\rangle \rho^{-}\left(p n ; J_{\alpha}\right)
\end{aligned}
$$

The ground state defined in (2.27) is more accurate that the BCS ground state $\left(a_{k}|B C S\rangle=0\right)$ since it contains terms with $0,4,8, \cdots$ quasiparticles [9]. Nevertheless, in the present model we approximate the initial ground state in the $(A, Z)$ nucleus by the BCS vacuum and the states $\left|J_{\alpha}\right\rangle$ in the intermediate $(A, Z \mp 1)$ nuclei as

$$
\begin{aligned}
\left|J_{\alpha}\right\rangle & =\sum_{p n} X_{p n J_{\alpha}} A^{\dagger}(p n J)|B C S\rangle, \\
H_{1}\left|J_{\alpha}\right\rangle & =\omega_{J_{\alpha}}\left|J_{\alpha}\right\rangle .
\end{aligned}
$$

This disadvantage of the present model is counteracted by the description that we make of the final states in the $(A, Z \mp 2)$ nuclei. That is, instead of the correlated $\left|0_{F}^{+}\right\rangle$ state defined in (2.26), we have

$$
\begin{aligned}
\left|\mathcal{J}_{f}^{+}\right\rangle & =\sum_{p_{1} p_{2} n_{1} n_{2} J_{n} J_{p}} Y_{\mathrm{p}_{1} \mathrm{p}_{2} \mathrm{~J}_{\mathrm{p}}, \mathrm{n}_{1} \mathrm{n}_{2} \mathrm{~J}_{\mathrm{n}} ; \mathcal{J}_{f}^{+}} \\
& \times\left|p_{1} p_{2} J_{p}, n_{1} n_{2} J_{n} ; \mathcal{J}^{+}\right\rangle_{A},
\end{aligned}
$$


where $\mathcal{J}^{+}=0^{+}, 2^{+}$and

$$
\begin{aligned}
& \left|p_{1} p_{2} J_{p}, n_{1} n_{2} J_{n} ; \mathcal{J}^{+}\right\rangle_{A} \\
= & {\left[\mathcal{A}^{\dagger}\left(p_{1} p_{2} J_{p}\right) \mathcal{A}^{\dagger}\left(n_{1} n_{2} J_{n}\right)\right]^{\mathcal{J}^{+}}|B C S\rangle, }
\end{aligned}
$$

are antisymmetrized and normalized two-proton-twoneutron quasiparticle states, with

$$
\begin{aligned}
\mathcal{A}^{\dagger}(a b J) & =N(a b) A^{\dagger}(a b J), \\
N(a b) & =\frac{1}{\sqrt{1+\delta_{a b}}},
\end{aligned}
$$

being normalized two-quasiparticle states.

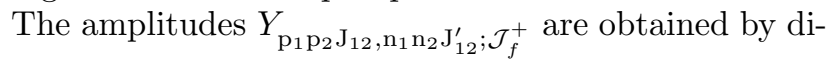
agonalizing the Hamiltonian [24, 25]

$$
\begin{aligned}
& H_{2}=H_{0}+H_{p n}+H_{n n}+H_{p p} \\
& H_{2}\left|\mathcal{J}_{f}^{+}\right\rangle=\omega_{\mathcal{J}_{f}^{+}}\left|\mathcal{J}_{f}^{+}\right\rangle
\end{aligned}
$$

in the basis (2.31) with $H_{n n}$ and $H_{p p}$ being neutronneutron and proton-proton interactions. Details on the evaluation of matrix elements of $\mathrm{H}_{2}$ can be found in References [24, 25]. However, our final results are different.

The matrix element of $H_{p n}$ for the odd-odd nucleus reads

$$
\begin{aligned}
& \left\langle B C S\left|A(n p J) H_{p n} A^{\dagger}\left(n^{\prime} p^{\prime} J\right)\right| B C S\right\rangle \\
= & G\left(n p n^{\prime} p^{\prime} J\right)\left(u_{p} u_{n} u_{p^{\prime}} u_{n^{\prime}}+v_{p} v_{n} v_{p^{\prime}} v_{n^{\prime}}\right) \\
+ & F\left(n p n^{\prime} p^{\prime} J\right)\left(u_{p} v_{n} u_{p^{\prime}} v_{n^{\prime}}+v_{p} u_{n} v_{p^{\prime}} u_{n^{\prime}}\right)
\end{aligned}
$$

where the functions $G$ and $F$ are defined in the standard way [26].

The matrix elements of $H_{p n}$ in the basis (2.31) are derived by employing the relation (1A-25) from [27]. We get

$$
\begin{aligned}
& \left\langle B C S\left|\left[\mathcal{A}^{\dagger}\left(n_{1} n_{2} J_{n}\right) \mathcal{A}^{\dagger}\left(p_{1} p_{2} J_{p}\right)\right]^{\mathcal{J} \dagger} H_{p n}\left[\mathcal{A}^{\dagger}\left(n_{1}^{\prime} n_{2}^{\prime} J_{n}^{\prime}\right) \mathcal{A}^{\dagger}\left(p_{1}^{\prime} p_{2}^{\prime} J_{n}^{\prime}\right)\right]^{\mathcal{J}}\right| B C S\right\rangle \\
= & \hat{J}_{n} \hat{J}_{p} \hat{J}_{n}^{\prime} \hat{J}_{p}^{\prime} N\left(n_{1} n_{2}\right) N\left(n_{1}^{\prime} n_{2}^{\prime}\right) N\left(p_{1} p_{2}\right) N\left(p_{1}^{\prime} p_{2}^{\prime}\right) \bar{P}\left(n_{1} n_{2} J_{n}\right) \bar{P}\left(p_{1} p_{2} J_{p}\right) \\
\times & \bar{P}\left(n_{1}^{\prime} n_{2}^{\prime} J_{n}^{\prime}\right) \bar{P}\left(p_{1}^{\prime} p_{2}^{\prime} J_{p}^{\prime}\right) \sum_{J_{1} J_{2}} \hat{J}_{1}^{2} \hat{J}_{2}^{2}\left\{\begin{array}{ccc}
n_{1} & n_{2} & J_{n} \\
p_{1} & p_{2} & J_{p} \\
J_{1} & J_{2} & \mathcal{J}
\end{array}\right\}\left\{\begin{array}{ccc}
n_{1}^{\prime} & n_{2}^{\prime} & J_{n}^{\prime} \\
p_{1}^{\prime} & p_{2}^{\prime} & J_{p}^{\prime} \\
J_{1} & J_{2} & \mathcal{J}
\end{array}\right\} \\
\times & \left\langle B C S\left|\mathcal{A}^{\dagger}\left(n_{1} p_{1} J_{1}\right) H_{p n} \mathcal{A}^{\dagger}\left(n_{1}^{\prime} p_{1}^{\prime} J_{1}\right)\right| B C S\right\rangle \delta_{p_{2}} p_{2}^{\prime} \delta_{n_{2} n_{2}^{\prime}}
\end{aligned}
$$

where the operator

$$
\bar{P}\left(p_{1} p_{2} J\right)=1+(-)^{p_{1}-p_{2}+J} P\left(p_{1} \leftrightarrow p_{2}\right),
$$

Finally, the matrix element of the neutron-neutron Hamiltonian $H_{n n}$ in the same basis is

exchanges the particles $p_{1}$ and $p_{2}$.

$$
\begin{aligned}
& \left\langle B C S\left|\left[\mathcal{A}^{\dagger}\left(n_{1} n_{2} J_{n}\right) \mathcal{A}^{\dagger}\left(p_{1} p_{2} J_{p}\right)\right]^{\mathcal{J} \dagger} H_{n n}\left[\mathcal{A}^{\dagger}\left(n_{1}^{\prime} n_{2}^{\prime} J_{n}^{\prime}\right) \mathcal{A}^{\dagger}\left(p_{1}^{\prime} p_{2}^{\prime} J_{p}^{\prime}\right)\right]^{\mathcal{J}}\right| B C S\right\rangle \\
= & \delta_{J_{p} J_{p}^{\prime}} \delta_{J_{n} J_{n}^{\prime}} \delta_{p_{1} p_{1}^{\prime}} \delta_{p_{2} p_{2}^{\prime}}\left\langle B C S\left|\mathcal{A}^{\dagger}\left(n_{1} n_{2} J_{n}\right) H_{n n} \mathcal{A}^{\dagger}\left(n_{1}^{\prime} n_{2}^{\prime} J_{n}\right)\right| B C S\right\rangle \\
= & \delta_{J_{p} J_{p}^{\prime}} \delta_{J_{n} J_{n}^{\prime}} \delta_{p_{1} p_{1}^{\prime}} \delta_{p_{2} p_{2}^{\prime}} N\left(n_{1} n_{2}\right) N\left(n_{1}^{\prime} n_{2}^{\prime}\right) \\
\times & {\left[\left(u_{n_{1}} u_{n_{2}} u_{n_{1}^{\prime}} u_{n_{2}^{\prime}}+v_{n_{1}} v_{n_{2}} v_{n_{1}^{\prime}} v_{n_{2}^{\prime}}\right) G\left(n_{1} n_{2} n_{1}^{\prime} n_{2}^{\prime} J_{n}\right)\right.} \\
+ & \left(u_{n_{1}} v_{n_{2}} u_{n_{1}^{\prime}} v_{n_{2}^{\prime}}+v_{n_{1}} u_{n_{2}} v_{n_{1}^{\prime}} u_{n_{2}^{\prime}}\right) F\left(n_{1} n_{2} n_{1}^{\prime} n_{2}^{\prime} J_{n}\right) \\
- & \left.(-1)^{n_{1}+n_{2}-J_{12}}\left(u_{n_{1}} v_{n_{2}} v_{n_{1}^{\prime}} u_{n_{2}^{\prime}}+v_{n_{1}} u_{n_{2}} u_{n_{1}^{\prime}} v_{n_{2}^{\prime}}\right) F\left(n_{2} n_{1} n_{1}^{\prime} n_{2}^{\prime} J_{n}\right)\right]
\end{aligned}
$$

and analogously for the proton-proton Hamiltonian $H_{p p}$.

The energies in the denominator $\mathcal{D}_{J_{\alpha}, \mathcal{J}_{f}}^{2 \nu^{ \pm}}$, defined by $(2.3)$, are

$$
E_{J_{\alpha}}^{\{ \pm 1\}}-E_{0^{+}}^{\{0\}}=\omega_{J_{\alpha}} \pm \lambda_{p} \mp \lambda_{n},
$$

$$
E_{\mathcal{J}_{f}}^{\{ \pm 2\}}-E_{0^{+}}^{\{0\}}=\omega_{\mathcal{J}_{f}} \pm 2 \lambda_{p} \mp 2 \lambda_{n}
$$

where $\lambda_{p}$ and $\lambda_{n}$ are the proton and neutron chemical potentials. Therefore, for both $2 \beta^{+}$and $2 \beta^{-}$-decays, they 
are

$$
\mathcal{D}_{J_{\alpha}, \mathcal{J}_{f}}^{2 \nu^{ \pm}} \equiv \mathcal{D}_{J_{\alpha}, \mathcal{J}_{f}}^{2 \nu}=\omega_{J_{\alpha}}-\frac{\omega_{\mathcal{J}_{f}}}{2}
$$

The lowest energies $E_{0_{f}^{+}}^{\{ \pm 2\}}$ are directly related with the $Q$-values for the $2 \beta^{-}$-decay $\left(Q_{2 \beta^{-}}\right)$and for the $2 e$-capture $\left(Q_{2 e}\right)$, defined as ${ }^{4}$

$$
\begin{aligned}
Q_{2 \beta^{-}} & =\mathcal{M}(Z, A)-\mathcal{M}(Z+2, A), \\
Q_{2 e} & =\mathcal{M}(Z, A)-\mathcal{M}(Z-2, A),
\end{aligned}
$$

where the $\mathcal{M}$ 's are the atomic masses. Namely,

$$
\begin{aligned}
Q_{2 \beta^{-}} & =E_{0^{+}}^{\{0\}}-E_{0_{1}^{+}}^{\{+2\}}=-\omega_{0_{1}^{+}}-2\left(\lambda_{p}-\lambda_{n}\right), \\
Q_{2 e} & =E_{0^{+}}^{\{0\}}-E_{0_{1}^{+}}^{\{-2\}}=-\omega_{0_{1}^{+}}+2\left(\lambda_{p}-\lambda_{n}\right) .
\end{aligned}
$$

Note that $Q_{2 e}-Q_{2 \beta^{-}}=4\left(\lambda_{p}-\lambda_{n}\right)$, and $Q_{2 e}+Q_{2 \beta^{-}}=$ $-2 \omega_{0_{1}^{+}}$.

To evaluate the one-body densities (2.4) and (2.8) we make use of [23, Eqs. (15.4)] to get

$$
\begin{aligned}
& \left(c_{p}^{\dagger} c_{\bar{n}}\right)_{J} \rightarrow u_{n} v_{p} A^{\dagger}(n p J), \\
& \left(c_{n}^{\dagger} c_{\bar{p}}\right)_{J} \rightarrow u_{p} v_{n} A^{\dagger}(p n J),
\end{aligned}
$$

which from (2.2) and (2.5) immediately yields

$$
\rho^{ \pm}\left(p n J_{\alpha}\right)=X_{p n J_{\alpha}}\left\{\begin{array}{l}
u_{n} v_{p} \\
u_{p} v_{n}
\end{array}\right\}
$$

and

$$
\left\langle J_{\alpha}^{+}\left\|\mathcal{O}_{J}^{ \pm}\right\| 0_{i}^{+}\right\rangle=\sum_{p n} X_{p n J_{\alpha}} O_{J}^{ \pm}(p n)
$$

with

$$
O_{J}^{ \pm}(p n)=O_{J}(p n)\left\{\begin{array}{l}
u_{n} v_{p} \\
u_{p} v_{n}
\end{array}\right\}
$$

The derivation of $\left\langle\mathcal{J}_{f}^{+}\left\|\mathcal{O}_{J}^{ \pm}\right\| J_{\alpha}^{+}\right\rangle$is more laborious and one gets

$$
\begin{aligned}
& \left\langle\mathcal{J}_{f}^{+}\left\|\mathcal{O}_{J}^{ \pm}\right\| J_{\alpha}^{+}\right\rangle=\hat{J} \hat{\mathcal{J}}_{f} \sum_{p n p^{\prime} n^{\prime} J_{p} J_{n}}(-)^{J_{p}+J_{n}} \hat{J}_{p} \hat{J}_{n} \\
\times & N\left(n n^{\prime}\right) N\left(p p^{\prime}\right) Y_{\mathrm{pp}^{\prime} J_{\mathrm{p}}, n n^{\prime} J_{\mathrm{n}} ; \mathcal{J}_{f}^{+}} \bar{P}\left(n n^{\prime} J_{n}\right) \bar{P}\left(p p^{\prime} J_{p}\right) \\
\times & \left\{\begin{array}{ccc}
p & p^{\prime} & J_{p} \\
n & n^{\prime} & J_{n} \\
J & J & \mathcal{J}
\end{array}\right\} X_{p^{\prime} n^{\prime} J_{\alpha}} O_{J}^{ \pm}(p n) .
\end{aligned}
$$

The densities $\rho^{ \pm}\left(p n ; J_{\alpha}^{\pi}, \mathcal{J}_{f}^{+}\right)$result immediately (2.7) and (2.46).

Making use of orthogonality and completeness of both basis $\left|J_{\alpha}\right\rangle$ and $A^{\dagger}(p n J)|B C S\rangle$ in (2.29), the relation (2.15) can be expressed in a more compact form. Namely as,

$$
\begin{aligned}
B_{J \mathcal{J}}^{\{ \pm 2\}} & =\hat{\mathcal{J}}^{2} \mid \sum_{p p^{\prime} n n^{\prime} J_{p} J_{n}}(-)^{J_{p}+J_{n}} \hat{J}_{p} \hat{J}_{n} N\left(n n^{\prime}\right) N\left(p p^{\prime}\right) \\
& \times Y_{\mathrm{pp}^{\prime} \mathrm{J}_{\mathrm{p}}, n n^{\prime} \mathrm{J}_{\mathrm{n}} ; \mathcal{J}_{f}^{+}} \bar{P}\left(n n^{\prime} J_{n}\right) \bar{P}\left(p p^{\prime} J_{p}\right) \\
& \times\left.\left\{\begin{array}{ccc}
p & p^{\prime} & J_{p} \\
n & n^{\prime} & J_{n} \\
J & J & \mathcal{J}
\end{array}\right\} O_{J}^{\mp}\left(p^{\prime} n^{\prime}\right) O_{J}^{\mp}(p n)\right|^{2}
\end{aligned}
$$

In this way, the transition strength turns out to be independent of the intermediate states.

It is important to emphasize that the permutation operators in the last two equations only act on the right side. The physical meaning of these permutations can be inferred from the diagram (b) in Fig. 1, where is graphically represented the DCE matrix element $\sum_{\alpha}\left\langle\mathcal{J}_{f}^{+}\left\|\mathcal{O}_{J}^{-}\right\| J_{\alpha}^{+}\right\rangle\left\langle J_{\alpha}^{+} \| \mathcal{O}_{J}^{-} \mid 0_{i}^{+}\right\rangle$. This quantity is used in the evaluation of both the $\beta \beta$-decay NME (2.9) and the DCE transition strengths (2.15), but the Eq. (2.47) is applicable only in the latter case.

Together with the NME $M^{2 \nu^{ \pm}}\left(\mathcal{J}_{f}^{+}\right)$given by $(2.1)$ and (2.9) with $M^{2 \nu^{ \pm}}\left(2_{f}^{+}\right) \equiv M_{G T}^{2 \nu^{ \pm}}\left(2_{f}^{+}\right)$, we will also evaluate the half-lives $\tau_{2 \nu^{ \pm}}^{\alpha}\left(\mathcal{J}_{f}^{+}\right)$for different $\alpha(=$ $\left.2 \beta^{-}, 2 \beta^{+}, e \beta^{+}, 2 e\right)$. This is done from

$$
\left[\tau_{2 \nu^{ \pm}}^{\alpha}\left(\mathcal{J}_{f}^{+}\right)\right]^{-1}=g_{A}^{4}\left|M^{2 \nu^{ \pm}}\left(\mathcal{J}_{f}^{+}\right)\right|^{2} G_{2 \nu}^{\alpha}\left(\mathcal{J}_{f}^{+}\right),
$$

i.e. the product of dimensionless axial vector coupling constant, $g_{A}$, common NME, $M^{2 \nu^{ \pm}}\left(\mathcal{J}_{f}^{+}\right)$, given in natural units $\left(\hbar=m_{e}=c=1\right)$, and different leptonic kinematics factors, $G_{2 \nu}^{\alpha}\left(\mathcal{J}_{f}^{+}\right)$, in $\mathrm{yr}^{-1}$. The last ones can be found in [28, Table II] for several nuclei of interest. (For the most recent computations of phase space factors see Refs. $[29,30]$.)

The excitation energies in the final nuclei are calculated from

$$
\mathcal{E}_{f}=E_{0_{f}^{+}}^{\{+2\}}-E_{0_{1}^{+}}^{\{+2\}} .
$$

It should be noted that, just as in the pn-QRPA model the excitation energies in the $(Z, A \pm 1)$ nuclei are the same, in the current model the excitation energies in the $(Z, A \pm 2)$ nuclei are the same.

Finally, the centroid energies of the DCE transition strengths are defined as

$$
\bar{E}_{J \mathcal{J}}^{\{ \pm 2\}}=\frac{\sum_{f} \mathcal{E}_{\mathcal{J}_{f}^{+}} B_{J \mathcal{J}_{f}}^{\{ \pm 2\}}}{S_{J \mathcal{J}}^{\{ \pm 2\}}} .
$$

\footnotetext{
$$
\begin{aligned}
& Q_{2 \beta^{+}}=\mathcal{M}(Z, A)-\mathcal{M}(Z-2, A)-4 m_{e}, \\
& Q_{\beta^{+} e}=\mathcal{M}(Z, A)-\mathcal{M}(Z-2, A)-2 m_{e} .
\end{aligned}
$$

${ }^{4}$ The $2 \beta^{+}$and $\beta^{+} e$ Q-values are:
} 

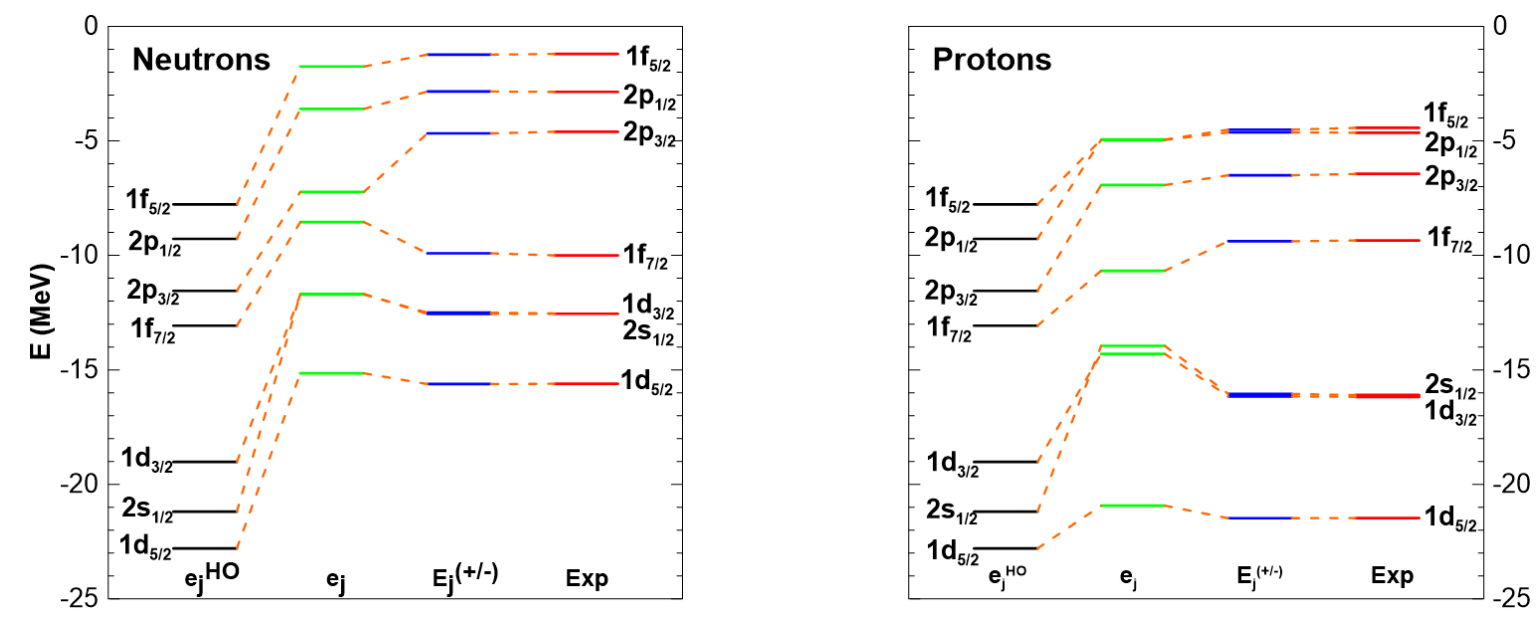

FIG. 2: (Color online) Mean field energies (in units of MeV) for neutrons (left panel) and for protons (right panel). In both cases are shown: (i) harmonic oscillator energies $e_{j}^{H O}$, (ii) adjusted single particle energies $e_{j}$, (iii) BCS energies relative to the Fermi level $\left(E_{j}^{( \pm)}\right)$, and (iv) experimental energies $\left(e_{j}^{e x p}\right)$.

\section{NUMERICAL RESULTS AND DISCUSSION}

The residual interaction is described by the $\delta$-force (in units of $\mathrm{MeV} \cdot \mathrm{fm}^{3}$ )

$$
V=-4 \pi\left(v^{s} P_{s}+v^{t} P_{t}\right) \delta(r),
$$

where $v^{s}$ and $v^{t}$ are the spin-singlet and spin-triplet parameters.

As usually, the pairing strengths for protons and neutrons, $v_{\text {pair }}^{s}(\mathrm{p})$ and $v_{\text {pair }}^{s}(\mathrm{n})$, are obtained from the fitting of the experimental pairing gaps.

In the numerical evaluations of the matrix elements $G\left(n p n^{\prime} p^{\prime} J\right), \quad F\left(n p n^{\prime} p^{\prime} J\right), \quad G\left(n_{1} n_{2} n_{1}^{\prime} n_{2}^{\prime} J_{n}\right)$, $F\left(n_{1} n_{2} n_{1}^{\prime} n_{2}^{\prime} J_{n}\right), \quad G\left(p_{1} p_{2} p_{1}^{\prime} p_{2}^{\prime} J_{p}\right), \quad$ and $F\left(p_{1} p_{2} p_{1}^{\prime} p_{2}^{\prime} J_{p}\right)$ of the Hamiltonians $H_{p n}, H_{p p}, H_{p n}$, given by Eqs. (2.32), (2.33) and (2.34), were used the same coupling constants.

To set the coupling constants in the $p h$-channel we use the energy behavior of the IAS and GTR [31] (see also Refs. $[32,33]$ ), with the results (in units of $\mathrm{MeV} \cdot \mathrm{fm}^{3}$ ): i) $v_{p h}^{s}=27$ and $v_{p h}^{t}=64$ for ${ }^{48} \mathrm{Ca}$, and ii) $v_{p h}^{s}=55$ and $v_{p h}^{t}=92$ for all nuclei.

For the coupling constants $v_{p p}^{s}$ and $v_{p p}^{t}$ within the $p p$ channel, we use values close to those obtained in Ref. [19] as a result of the partial restoration of the spin-isospin $\mathrm{SU}(4)$ symmetry (PSU4SR). More precisely, this procedure yields $s_{\text {sym }} \cong t_{\text {sym }} \cong 1$ for the ratios

$$
s=\frac{v_{p p}^{s}}{\bar{v}_{\text {pair }}^{s}}, \quad t=\frac{v_{p p}^{t}}{\bar{v}_{\text {pair }}^{s}},
$$

where $\bar{v}_{\text {pair }}^{s}=\left(v_{\text {pair }}^{s}(\mathrm{p})+v_{\text {pair }}^{s}(\mathrm{n})\right) / 2 .{ }^{5}$

Moreover, instead of using the bare value $g_{A}=1.27$ for the axial-vector coupling constant [34], we use an effective value $g_{A}=1.0^{6}$. Still smaller values for $g_{A}$ have been used in the literature [36].

\section{A. Single particle space}

The $\mathrm{DBD}^{-}{ }^{48} \mathrm{Ca} \rightarrow{ }^{48} \mathrm{Ti}$ is a rather unique case, since ${ }^{48} \mathrm{Ca}$ is a double closed nuclei, and we can make use of the experimental spe $e_{j}^{e x p}$. All they were taken from the binding and excitation energies, weighted with spectroscopic factors, of odd-mass neighboring nuclei: ${ }^{47} \mathrm{Ca}$ and ${ }^{49} \mathrm{Ca}$ for neutrons, and ${ }^{47} \mathrm{~K}$ and ${ }^{49} \mathrm{Sc}$ for protons. They are listed in Fig. 2 and are those from [38, Table II], except for the proton $f_{5 / 2}$ spe, which is estimated from the proton $f_{5 / 2}-f_{7 / 2}$ splitting given in Ref. [39]. We need this level to saturate both the SCE and DCE sum rules. Once this has been done the spe $e_{j}^{e x p}$ have been used in two different ways:

\footnotetext{
${ }^{5}$ Within PSU4SR $s$ and $t$ are determined from the condition that the strengths $S_{F}^{+} \equiv S_{0}^{\{-1\}}$ and $S_{G T}^{+} \equiv S_{1}^{\{-1\}}$ become minimal (see [19, Fig. 1]).

6 This quenching is frequently attributed to the $\Delta$-hole polarization effect on the axial-vector coupling constant [32]. Recently has been presented an explanation of the quenching of $g_{A}$ within the context of effective field theories [35].
} 
1. The following steps are done in handling the BCS equations $[12,37]$ :

a) The BCS energies relative to the Fermi level $\lambda$,

$$
E_{j}^{( \pm)}= \pm E_{j}+\lambda
$$

are introduced, where the positive (negative) sign is adopted if the corresponding single-particle state is a particle (hole)-state.

b) It is assumed that neutron and proton Fermi levels $\lambda_{n}$, and $\lambda_{p}$ lay between $j_{n}=2 p_{3 / 2}-1 f_{7 / 2}$ and $j_{p}=1 f_{7 / 2}-2 s_{3 / 2}$ states respectively, and that all states above $\lambda$ are pure quasi-particle excitations $E_{j}^{(+)}$and all states below $\lambda$ are pure quasi-hole excitations $E_{j}^{(-)}$.

c) Starting from a set of harmonic oscillator energies $e_{j}^{H O}$, the energies $E_{j}^{( \pm)}$are adjusted to the experimental spectra $e_{j}^{\exp }$ by means of a $\chi^{2}$ search varying the strengths $v_{s}^{\text {pair }}$ and the bare spe $e_{j}$ which that appear in the BCS gap equations (2.24).

All this procedure is illustrated in Fig. 2.

2. For the sake of completeness the pairing parameters $v_{\text {pair }}^{s}(\mathrm{p})$ and $v_{\text {pair }}^{s}(\mathrm{n})$ were fixed in the standard manner [49]. That is, by fitting the experimental pairing gaps to the calculated pairing gaps $\Delta_{j}$, given by [27, Eq. (2.96)], with $j=1 f_{7 / 2}$ for neutrons and $j=2 s_{1 / 2}$ for protons.

The most relevant difference between the spe $e_{j}^{e x p}$ and $e_{j}$ is the disappearance of the energy gap between the holes and the particles in the last case. The resulting parameters $v_{s}^{\text {pair }}$, and $\lambda$ are given in the Table I for the two sets of spe $e_{j}$ and $e_{j}^{e x p}$. The quasiparticle energies $E_{j}^{( \pm)}$with $e_{j}^{\exp }$ are obviously slightly different from those shown in Fig. 2.

TABLE I: Results for the BCS coupling constants and Fermi levels. All notation is explained in the text. The $\lambda$ 's are given in units of $\mathrm{MeV}$, and the couplings $v_{s}^{\text {pair }}$ is in units of $\mathrm{MeV} \cdot \mathrm{fm}^{3}$.

\begin{tabular}{c|c|cc|cc}
\hline \hline Nuclei & spe & $v_{s}^{\text {pair }}(n)$ & $v_{s}^{\text {pair }}(p)$ & $\lambda_{n}$ & $\lambda_{p}$ \\
\hline \multirow{2}{*}{${ }^{48} \mathrm{Ca}$} & $e_{j}^{\text {exp }}$ & 31.45 & 34.77 & -6.587 & -13.000 \\
& $e_{j}$ & 25.20 & 28.35 & -7.091 & -12.702 \\
\hline${ }^{96} \mathrm{Ru}$ & & 33.20 & 38.91 & -8.412 & -5.663 \\
\hline \hline
\end{tabular}

In the ${ }^{96} \mathrm{Ru}$ nucleus, the neutron and proton shells are both open and its energy spectra is clearly rotational. This fact gives rise to a strong interplay between collective and single-particle degrees of freedom in the low energy spectra of the neighboring odd-mass nuclei ${ }^{95} \mathrm{Ru}$, ${ }^{97} \mathrm{Ru},{ }^{95} \mathrm{Tc}$, and ${ }^{97} \mathrm{Ru}$. For instance, it is very likely that the ground state $5 / 2^{+}$in ${ }^{95} \mathrm{Ru}$ is a consequence of the $j-1$ anomaly in the three neutron cluster $\left(1 g_{7 / 2}\right)^{3}[45,46]$.
Such a complex nuclear structure inhibits us to determine the appropriate spe spectra and the pairing interaction strengths from experimental energy spectra, as it was done in the case of ${ }^{48} \mathrm{Ca}$.

TABLE II: Neutron and proton spe for ${ }^{96} \mathrm{Ru}$, which were obtained in the way explained in the text, together with the resulting quasiparticle energies (3.3). All notation is explained in the text. The energies are given in units of $\mathrm{MeV}$, and the couplings in units of $\mathrm{MeV} \cdot \mathrm{fm}^{3}$.

\begin{tabular}{c|rr|rr}
\hline \multirow{2}{*}{ level } & \multicolumn{2}{|c|}{ Neutrons } & \multicolumn{2}{c}{ Protons } \\
\cline { 2 - 5 } & $e_{j}$ & $E_{j}^{( \pm)}$ & $e_{j}$ & $E_{j}^{( \pm)}$ \\
\hline $3 s_{1 / 2}$ & -5.396 & -5.1817 & 4.916 & 5.006 \\
$2 d_{3 / 2}$ & -5.236 & -4.9834 & 4.792 & 4.897 \\
$1 g_{7 / 2}$ & -6.878 & -6.5267 & 2.308 & 2.502 \\
$2 d_{5 / 2}$ & -7.401 & -10.0514 & 2.493 & 2.627 \\
$1 g_{9 / 2}$ & -14.401 & -14.5004 & -5.424 & -7.448 \\
$2 p_{1 / 2}$ & -17.802 & -17.9127 & -7.718 & -8.478 \\
$2 p_{3 / 2}$ & -19.389 & -19.4838 & -9.295 & -9.773 \\
\hline \hline
\end{tabular}

We used instead the spe provided by N. Paar [47], which were calculated in the relativistic HartreeBogoliubov model, as outlined in Ref. [48]. They are shown in Table II, together with the resulting quasiparticle energies (3.3), which were obtained following procedure 2 in the case of ${ }^{48} \mathrm{Ca}$. This implies to fit the calculated pairing gaps $\Delta_{j}$, with $j=1 g_{7 / 2}$ for neutrons and $j=1 g_{9 / 2}$ for protons, to the experimental ones. The similarity between the spe $e_{j}$ and the quasiparticle energies $E_{j}^{( \pm)}$is remarkable. The corresponding pairing parameters $v_{s}^{\text {pair }}$ and chemical potentials $\lambda$ are listed in Table I.

It is important to note that in ${ }^{48} \mathrm{Ca}$ it is $\lambda_{n}>\lambda_{p}$ while in ${ }^{76} \mathrm{Ru}$ this difference is $\lambda_{n}<\lambda_{p}$. We will soon see that this fact is decisive with respect to the $Q$-values of DBD. More precisely, this will explain why $\mathrm{DBD}^{-}$occurs in ${ }^{48} \mathrm{Ca}$ and $\mathrm{DBD}^{+}$occurs in ${ }^{76} \mathrm{Ru}$.

Our method of calculation is similar in several aspects to that used in the SM in the Refs. [6, 7]. In fact, the illustration of their calculations, made in Fig. 1 of that reference, is also valid in our case. The biggest difference between the two models, in addition to the residual interactions that were used, arises from the size of the configuration spaces of the final states $\mathcal{J}_{f}^{+}$. We have $6640^{+}$states and $2.4702^{+}$states, while Auerbach and Bui Minh Loc [7] have, in their evaluation of double charge-exchange GT strength ${ }^{48} \mathrm{Ca} \rightarrow{ }^{48} \mathrm{Ti}$, the quantity of 14.177 and 61.953 final states in the $f p$-space, respectively. In the case of the ${ }^{96} \mathrm{Ru} \rightarrow{ }^{96} \mathrm{Mo}$ decay, we also have $6640^{+}$states, but $2.5832^{+}$states. $^{7}$

${ }^{7}$ See also our Fig. 1, where the difference with the standard pn- 

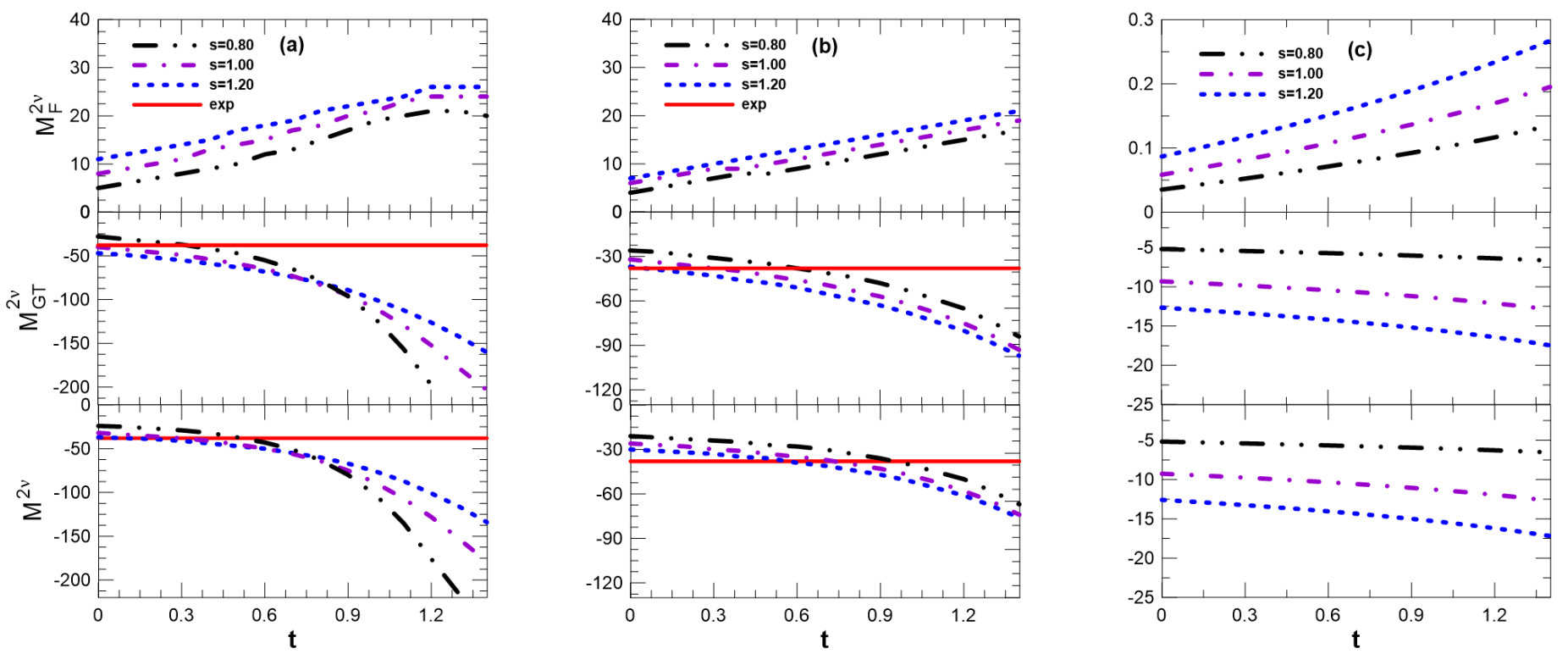

FIG. 3: Calculated NME $M_{F}^{2 \nu}, M_{G T}^{2 \nu}$ and $M^{2 \nu}$ for the ground $0^{+}$state (in natural units $\times 10^{-3}$ ), as a function of the $p p$ parameters $t$ and $s$ for ${ }^{48} \mathrm{Ti}$ for: a) ${ }^{48} \mathrm{Ca}$ with spe $e_{j}^{e x p}$, b) ${ }^{48} \mathrm{Ca}$ with spe $e_{j}$, and c) ${ }^{96} \mathrm{Ru}$. The experimental value for ${ }^{48} \mathrm{Ti}$ $\left|M^{2 \nu}\left(0_{1}^{+}\right)\right|=(38 \pm 3) \times 10^{-3}$ is also indicated by the red line, whose width represents the experimental error.

\section{B. Nuclear Matrix Elements}

We calculate simultaneously the NMEs $M_{F}^{2 \nu}\left(0_{f}^{+}\right)$, $M_{G T}^{2 \nu}\left(0_{f}^{+}\right), M^{2 \nu}\left(0_{f}^{+}\right)$, and $M_{G T}^{2 \nu}\left(2_{f}^{+}\right)$for all above mentioned $\mathcal{J}_{f}^{+}$final states with the following three sets of $p p$ parameters:

$$
\begin{array}{lll}
T 1: & s=0.80, & t=0.80, \\
T 2: & s=0.80, & t=1.00, \\
T 3: & s=1.00, & t=1.00 .
\end{array}
$$

In the upper part of Table III are shown the results for the ${ }^{48} \mathrm{Ca} \rightarrow{ }^{48} \mathrm{Ti}$ decays to the ground state $0_{1}^{+}$, and the first excited $0_{2}^{+}$and $2_{1}^{+}$states in ${ }^{48} \mathrm{Ti}$ nucleus, for the two sets of spe $e_{j}$ listed in Table I. The agreement between the calculated and measured results for $M^{2 \nu}\left(0_{1}^{+}\right)$can be considered satisfactory (in particular with the spe $e_{j}$ ) in view of the fact that all the nuclear parameters in the $p p$ and $p h$-channels are so to say fixed, both for identical particles and for different particles. We hope that in the next future the NMEs $M^{2 \nu}\left(0_{2}^{+}\right)$and $M_{G T}^{2 \nu}\left(2_{1}^{+}\right)$will also be measured. For both $0^{+}$levels the F-NME is relatively small compared to the GT one, but in no way it can be neglected. As seen in the Eq. (2.1), these two NMEs always interfere destructively. ${ }^{8}$

QRPA calculation of the NME is illustrated graphically

${ }^{8}$ It is interesting to note that $M_{F}$ is often omitted in the calcula-
The NME $\left|M^{2 \nu}\left(0_{1}^{+}\right)\right|$in ${ }^{48} \mathrm{Ca}$ has been calculated many times, but there are only very few theoretical studies of $M^{2 \nu}\left(0_{2}^{+}\right)$and $M_{G T}^{2 \nu}\left(2_{1}^{+}\right)$. As far as we know, the first one has been evaluated only in Ref. [41] and the second one in Refs.[17, 41] (see also Ref. [42]). These results, as well as those for NME $\left|M^{2 \nu}\left(0_{1}^{+}\right)\right|$, are confronted with our results in Table III. It should be pointed out that in the just mentioned studies have not been considered the contributions of F-NMEs $M_{F}^{2 \nu}\left(0_{\alpha=1,2}^{+}\right)$. Therefore, strictly speaking their results for $\left|M^{2 \nu}\left(0_{\alpha=1,2}^{+}\right)\right|$should be compared with ours $\left|M_{G T}^{2 \nu}\left(0_{\alpha=1,2}^{+}\right)\right|$.

In the lower part of Table III are shown the NMEs for the ${ }^{96} \mathrm{Ru} \rightarrow{ }^{96}$ Mo decay to final states $\mathcal{J}^{\pi}=0_{1}^{+}, 0_{2}^{+}$, and $2_{1}^{+}$. The results of previous calculations $[10,50-$ 53] are also shown. It is noticeable that the differences between our three calculations are much smaller than the differences with all the other works.

The strong dependence of the NME within the pnQRPA model with respect to the isoscalar $p p$ parameter $t$ is well known and is often discussed. Therefore, it could be interesting to analyze that dependence in the current model. This is done in Fig. 3 showing the NMEs $M_{F}^{2 \nu}$, $M_{G T}^{2 \nu}$ and $M^{2 \nu}$ for the ground $0^{+}$state in ${ }^{48} \mathrm{Ca}$ and ${ }^{96} \mathrm{Ru}$, as a function of the $p p$ parameters $t$ and $s$. The experimental value of $\left|M^{2 \nu}\left(0_{1}^{+}\right)\right|$is also drawn. It can be concluded that within the present model such dependence is

tions, simply invoking isospin conservation. 
TABLE III: Calculated and measured NME (in natural units $\left.\times 10^{-3}\right)$ for $2 \nu$-DBDs of ${ }^{48} \mathrm{Ca}\left(M^{2 \nu}\left(\mathcal{J}_{f}^{+}\right) \equiv M^{2 \nu^{-}}\left(\mathcal{J}_{f}^{+}\right)\right)$and ${ }^{96} \mathrm{Ru}\left(M^{2 \nu}\left(\mathcal{J}_{f}^{+}\right) \equiv M^{2 \nu^{+}}\left(\mathcal{J}_{f}^{+}\right)\right)$to ground state $0_{1}^{+}$, and first excited $0_{2}^{+}$and $2_{1}^{+}$states in final nuclei ${ }^{48} \mathrm{Ti}$ and ${ }^{96} \mathrm{Mo}$, respectively. Results from Refs.[17, 41] for ${ }^{48} \mathrm{Ca}$ and from Refs.[10, 50-53] for ${ }^{96} \mathrm{Ru}$, as well the experimental value for ${ }^{48} \mathrm{Ca}$ [3], are also shown in same units.

\begin{tabular}{|c|c|c|c|c|c|}
\hline \multicolumn{6}{|c|}{${ }^{48} \mathrm{Ca}$} \\
\hline par & $M_{F}^{2 \nu}\left(0_{1}^{+}\right)$ & $M_{G T}^{2 \nu}\left(0_{1}^{+}\right)$ & $\left|M^{2 \nu}\left(0_{1}^{+}\right)\right|$ & $\left|M^{2 \nu}\left(0_{2}^{+}\right)\right|$ & $\left|M^{2 \nu}\left(2_{1}^{+}\right)\right|$ \\
\hline$e_{j}$ & & & & & \\
\hline $\mathrm{T} 1$ & 11 & -44 & 33 & 18 & 0.98 \\
\hline $\mathrm{T} 2$ & 13 & -53 & 40 & 22 & 1.08 \\
\hline $\mathrm{T} 3$ & 15 & -62 & 47 & 15 & 0.69 \\
\hline$e_{j}^{e x p}$ & & & & & \\
\hline $\mathrm{T} 1$ & 15 & -78 & 63 & 5.0 & 0.53 \\
\hline $\mathrm{T} 2$ & 19 & -122 & 103 & 23 & 0.60 \\
\hline T3 & 21 & -110 & 89 & 34 & 0.33 \\
\hline$[17]$ & & & 22 & & 120 \\
\hline$[41]$ & & & 28 & 26 & 1.62 \\
\hline Exp. & & & $38 \pm 3$ & & \\
\hline \multicolumn{6}{|c|}{${ }^{96} \mathrm{Ru}$} \\
\hline $\mathrm{T} 1$ & 0.08 & -5.9 & 5.8 & 55.6 & 0.55 \\
\hline $\mathrm{T} 2$ & 0.10 & -6.1 & 6.0 & 63.6 & 0.68 \\
\hline T3 & 0.15 & -11.5 & 11.3 & 26.8 & 0.63 \\
\hline$[10]$ & & & $415-1437$ & $492-1554$ & $0.1-8.4$ \\
\hline$[50]$ & & & 251 & & \\
\hline$[51]$ & & & 101 & & \\
\hline$[52]$ & & & 54 & & \\
\hline$[53]$ & -0 & 2170 & 2170 & 50 & \\
\hline
\end{tabular}

only moderate. The same statement is valid also for all remaining $2 \nu \mathrm{NMEs}$.

It is also well known that the relatively small values of the NME in the pn-QRPA model come from the destructive interference between forward and backward going contributions [19]. That is, trough the ground state correlations (GSC). The quenching mechanism is different in the current model, and it is the consequence of the interplay between seniority-zero and seniority-four configurations in the final states. For example, in the case of ${ }^{48} \mathrm{Ca}$, within the space $e_{j}$ and with force parameters $\mathrm{T} 1$, the $\mathrm{NME} M^{2 \nu}$ for the three lowest $0^{+}$ states are: $-0.033,-0.018,-0.043$. While, when only the seniority-zero configurations are considered, one gets: $-0.074,-0.028,-0.090$ respectively, which confirms the above statement.

\section{Half-lives}

The half-lives are evaluated trivially from (2.47) using the NME and leptonic kinematic factors $G_{2 \nu}^{\alpha}\left(\mathcal{J}_{f}^{+}\right)$. De-
TABLE IV: Calculated half-lives $\tau_{2 \nu}^{2 \beta^{-}}\left(\mathcal{J}_{f}^{+}=0_{1}^{+}, 0_{2}^{+}, 2_{1}^{+}\right)$in units of $y r$ for the $2 \nu$-DBD ${ }^{48} \mathrm{Ca} \rightarrow{ }^{48} \mathrm{Ti}$, with spe $e_{j}$ the $p p$ parameters set and $g_{A}=1$ are shown and confronted with previous calculations and experiments. In Eq. (2.47) are used the $G_{2 \nu}^{\alpha}\left(\mathcal{J}_{f}^{+}\right)$factors from Ref. [43] for the levels $0_{1}^{+}(=$ $\left.1.56 \times 10^{-17} \mathrm{yr}^{-1}\right)$ and $0_{2}^{+}\left(=3.63 \times 10^{-22} \mathrm{yr}^{-1}\right)$, and from Ref. [44] for $2_{1}^{+}\left(=4.41 \times 10^{-18} \mathrm{yr}^{-1}\right)$.

\begin{tabular}{c|c|c|c}
\hline \hline & $0_{1}^{+}$ & $0_{2}^{+}$ & $2_{1}^{+}$ \\
\hline $\mathrm{T} 1$ & $5.91 \times 10^{19}$ & $8.51 \times 10^{24}$ & $2.36 \times 10^{23}$ \\
\hline $\mathrm{T} 2$ & $4.02 \times 10^{19}$ & $5.70 \times 10^{24}$ & $1.94 \times 10^{23}$ \\
\hline $\mathrm{T} 3$ & $2.91 \times 10^{19}$ & $1.23 \times 10^{25}$ & $4.76 \times 10^{23}$ \\
\hline Ref. [17] & & & $1.72 \times 10^{24}$ \\
\hline Ref. [41] & $3.3 \times 10^{19}$ & & $8.5 \times 10^{23}$ \\
\hline Exp [3] & $\left(4.4_{-0.5}^{+0.6}\right) \times 10^{19}$ & & \\
\hline \hline
\end{tabular}

TABLE V: Calculated half-lives $\tau_{2 \nu^{ \pm}}^{\alpha}\left(0_{1}^{+}\right)$in units of $y r$ for the $2 \nu$-DBD ${ }^{96} \mathrm{Ru} \rightarrow{ }^{96} \mathrm{Mo}$, with the $p p$ parameter set $\mathrm{T} 1$ and $g_{A}=1$ are shown and confronted with previous calculations and experiments. In Eq. (2.47) are used the $G_{2 \nu}^{\alpha}\left(\mathcal{J}_{f}^{+}\right)$factors from Ref. [16] for the channels $2 \beta^{+}\left(=1.080 \times 10^{-26} \mathrm{yr}^{-1}\right)$, $\beta^{+} e\left(=0.454 \times 10^{-21} \mathrm{yr}^{-1}\right)$, and $e e\left(=2.740 \times 10^{-21} \mathrm{yr}^{-1}\right)$.

\begin{tabular}{c|c|c|c|c|c}
\hline \hline$\alpha$ & present & {$[50]$} & {$[51]$} & {$[52]$} & {$[53]$} \\
\hline \hline $2 \beta^{+}$ & $2.8 \times 10^{30}$ & $5.8 \times 10^{26}$ & & $3.485 \times 10^{28}$ & $1.22 \times 10^{16}$ \\
\hline$\beta^{+} e$ & $6.5 \times 10^{25}$ & $1.2 \times 10^{22}$ & $8.6 \times 10^{22}$ & $9.100 \times 10^{23}$ & $3.78 \times 10^{20}$ \\
\hline$e e$ & $1.1 \times 10^{25}$ & $2.1 \times 10^{21}$ & $1.4 \times 10^{22}$ & $1.628 \times 10^{23}$ & $3.11 \times 10^{17}$ \\
\hline \hline
\end{tabular}

spite this, we present some of them only for the sake of completeness.

In Table IV are compared our results for the half-lives $\tau_{2 \nu^{-}}^{2 \beta^{-}}\left(\mathcal{J}_{f}^{+}=0_{1}^{+}, 0_{2}^{+}, 2_{1}^{+}\right)$in ${ }^{48} \mathrm{Ca}$ evaluated with spe $e_{j} p p$ parameters set with the previous ones.

The major difference appears for the $2_{1}^{+}$level.

The same is done in Table $\mathrm{V}$ for the half-lives $\tau_{2 \nu^{+}}^{\alpha}\left(0_{1}^{+}\right)$ in ${ }^{96} \mathrm{Ru}$ for different channels $\alpha=2 \beta^{+}, \beta^{+} e$, and $e e$. The experimental limits are: $\tau_{2 \nu^{+}}^{2 \beta^{+}}\left(0_{1}^{+}\right) \geq 1.4 \times 10^{20} \mathrm{yr}$ and $\tau_{2 \nu^{+}}^{\beta^{+} e}\left(0_{1}^{+}\right) \geq 0.8 \times 10^{20}$ yr [54].

\section{D. $Q$-values and Energy Spectra}

Before starting with the discussion of $Q$-values, it is convenient to remember that a physical phenomenon is allowed only when this quantity is positive.

In Table VI are confronted the experimental data with our results for the $Q_{\beta \beta}$ and $Q_{2 e}$ values in the DCE processes: a) ${ }^{48} \mathrm{Ca} \rightarrow{ }^{48} \mathrm{Ti}$ and ${ }^{48} \mathrm{Ca} \rightarrow{ }^{48} \mathrm{Ar}$, and b) ${ }^{96} \mathrm{Ru}$ $\rightarrow{ }^{96} \mathrm{Mo}$ and ${ }^{96} \mathrm{Ru} \rightarrow{ }^{96} \mathrm{Pl}$. One sees that the model is capable of reproducing not only the signs of the $Q$-values, but also their magnitudes, without having to modify the 

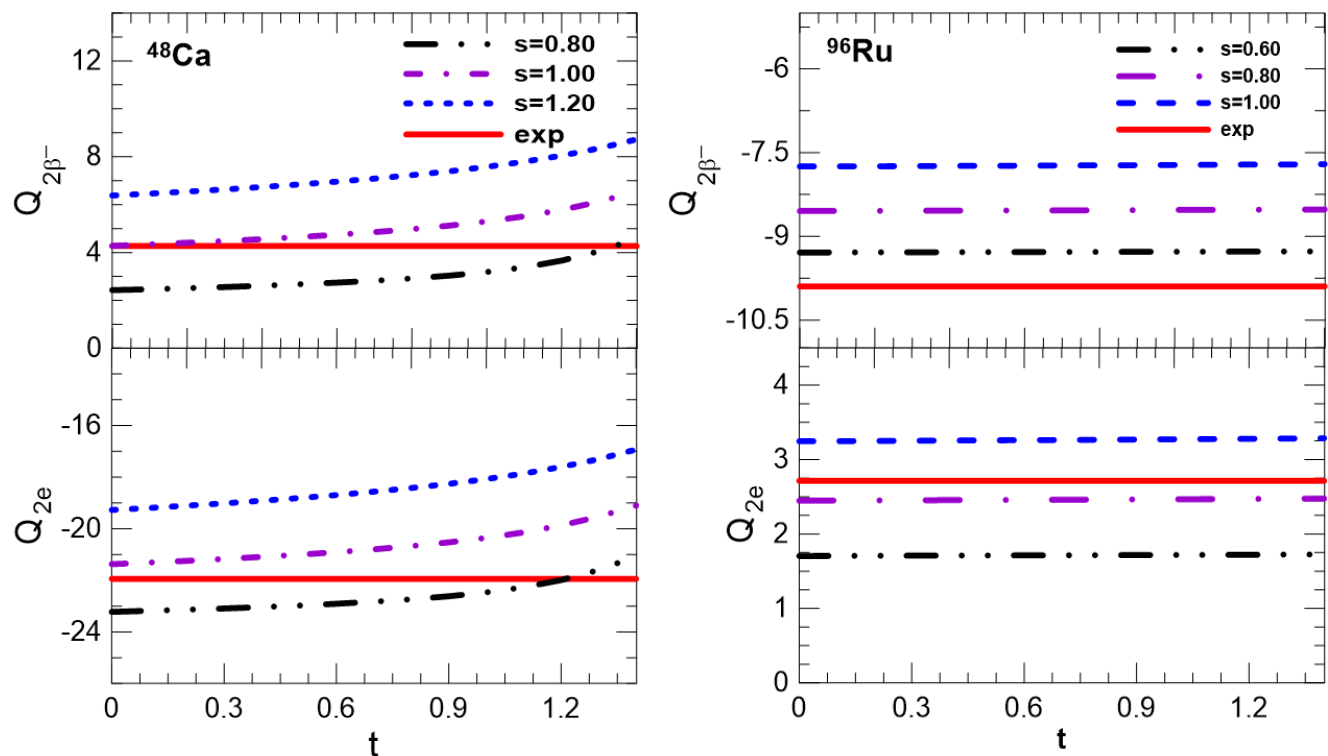

FIG. 4: Calculated $Q_{2 \beta^{-}}$and $Q_{2 e}$ values in ${ }^{48} \mathrm{Ca}$ within the spe space $e_{j}^{e x p}$ (left panel) and ${ }^{96} \mathrm{Ru}$ (right panel), as a function of $p p$ parameters $t$ and $s$. The experimental $Q$-values are also shown.

TABLE VI: Calculated $Q_{\beta \beta}$ and $Q_{2 e}$ values (in units of $\mathrm{MeV}$ ) with the three sets of $p p$ parameters $\mathrm{T} 1, \mathrm{~T} 2$ and $\mathrm{T} 3$ and for the DCE processes: a) ${ }^{48} \mathrm{Ca} \rightarrow{ }^{48} \mathrm{Ti}$ and ${ }^{48} \mathrm{Ca} \rightarrow{ }^{48} \mathrm{Ar}$, and b) ${ }^{96} \mathrm{Ru} \rightarrow{ }^{96} \mathrm{Mo}$ and ${ }^{96} \mathrm{Ru} \rightarrow{ }^{96} \mathrm{Pl}$, are confronted with the experimental ones.

\begin{tabular}{|c|c|c|c|}
\hline \multicolumn{4}{|c|}{${ }^{48} \mathrm{Ca}$} \\
\hline & par & $Q_{\beta \beta}$ & $Q_{2 e}$ \\
\hline \multirow{3}{*}{$e_{j}^{e x p}$} & $\mathrm{~T} 1$ & 2.919 & -22.734 \\
\hline & $\mathrm{T} 2$ & 3.185 & -22.468 \\
\hline & T3 & 5.300 & -20.353 \\
\hline \multirow{3}{*}{$e_{j}$} & $\mathrm{~T} 1$ & 5.151 & -17.295 \\
\hline & $\mathrm{T} 2$ & 5.232 & -17.214 \\
\hline & T3 & 6.710 & -15.736 \\
\hline Exp & & 4.268 & -21.943 \\
\hline \multicolumn{4}{|c|}{${ }^{96} \mathrm{Ru}$} \\
\hline \multirow{3}{*}{$e_{j}$} & $\mathrm{~T} 1$ & -8.532 & 2.460 \\
\hline & $\mathrm{T} 2$ & -8.527 & 2.465 \\
\hline & T3 & -7.721 & 3.271 \\
\hline Exp & & -9.896 & 2.714 \\
\hline
\end{tabular}

parameters of the model. This is very comforting! In addition, it seems that the model "knows" what type of DCE decay can occur in a given nucleus.

The nature of $Q$-value is dominantly determined by the proton and neutron pairing mean fields, as seen from (2.41) or, more precisely, from the relation $Q_{2 e}-Q_{2 \beta^{-}}=$ $4\left(\lambda_{n}-\lambda_{p}\right)$. The dependence on the residual interaction is rather weak and takes place through the ground state energy $\omega_{0_{1}^{+}}$in residual nuclei, as $Q_{2 e}+Q_{2 \beta^{-}}=-2 \omega_{0_{1}^{+}}$. More details on how the $Q$-values depend on the $p p$ coupling constants are shown in the Fig. 4.

As we stated before, in the same way that the pnQRPA model [2] predicts identical energy spectra for odd-odd nuclei $(A, Z \pm 1)$, the present model predicts identical excitation energies in even-even nuclei $(A, Z \pm$ 2 ). This is obviously not realistic due to the large neutron excess.

It is pertinent to mention here that the use of particlenumber-projection can become very important when working with the BCS mean-field [55]. Without a doubt, through this method different energy spectra are obtained in nuclei for which the number of protons is different.

But, despite the above mentioned handicap, the calculated excitation energies of the $0^{+}$and $2^{+}$states in ${ }^{48} \mathrm{Ti}$ are consistent with data, as shown in Fig. 5, where we give the calculated spectra for the two spe spaces and the $p p$ parameterizations $\mathrm{T} 1$ and $\mathrm{T} 3$. Because of the size of the $Q_{2 \beta^{-}-\text {value }}(=4.268 \mathrm{MeV})$, the $2 \beta^{-}$-decays are energetically possible for all states, except for $0_{4}^{+}$. We have evaluated the NMEs for all these states, but we do not consider necessary to present them here. It should also be said that we have not found in the literature any detailed calculation of the ${ }^{48} \mathrm{Ti}$ low energy spectrum to compare with ours. Finally, the ${ }^{96}$ Mo energy spectrum is not well explained by the current model and, therefore, 


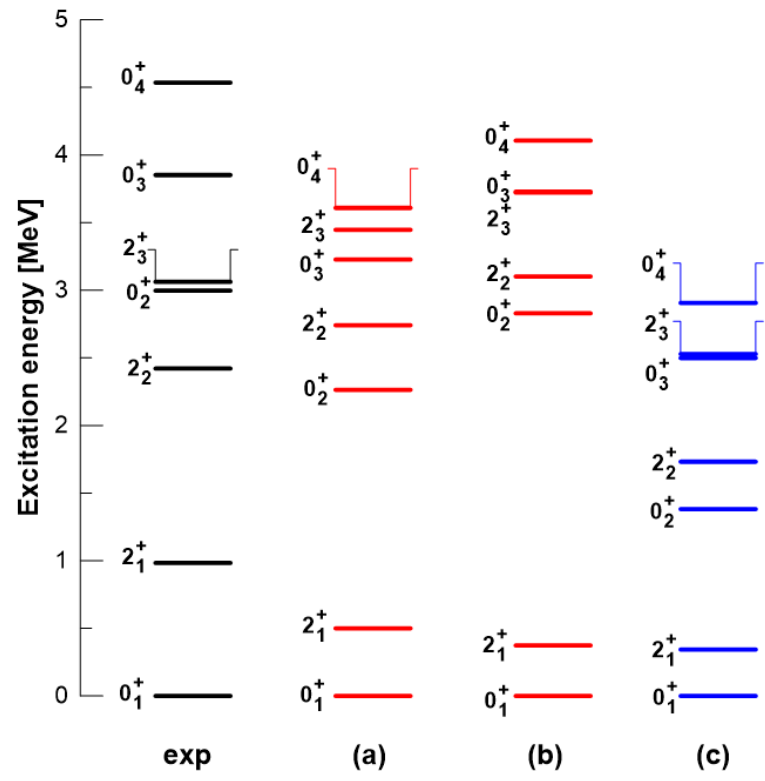

FIG. 5: Measured excitation energies in ${ }^{48} \mathrm{Ti}$ are compared with the calculations: (a) and (b) with the spe $e_{j}^{e x p}$ and $p p$ parametrization $\mathrm{T} 1$ and $\mathrm{T} 3$ respectively, and (c) with the spe $e_{j}$ and $p p$ parametrization $\mathrm{T} 1$.

will not be discussed here.

\section{E. Double-Charge-Exchange Strengths and their Sum Rules}

In Table VII are displayed the results for the DCE transition strengths $S_{J \mathcal{J}}^{\{ \pm 2\}}$ given by (2.15), both for Fermi $(J=0 ; \mathcal{J}=0)$ and Gamow-Teller $(J=1 ; \mathcal{J}=0,2)$. The corresponding sum rules $S_{J \mathcal{J}}^{\{2\}}$ calculated from (2.16) are also shown, and confronted with the predicted sum rules $\mathrm{S}_{J \mathcal{J}}^{\{2\}}$ given by $(2.17),(2.18)$, and (2.19).

In addition, to know the locations of the DCE resonances, we have evaluated the centroid energy (2.46), and to get an idea of the magnitudes of the DBD, the strengths $B_{J J_{1}}^{\{+2\}}\left(B_{J J_{1}}^{\{-2\}}\right)$ going to the levels $0_{1}^{+}$and $2_{1}^{+}$ in final ${ }^{48} \mathrm{Ti}\left({ }^{96} \mathrm{Mo}\right)$ nucleus are explicitly given.

All calculations related to DCE transition strengths were performed for the three sets of $p p$ parameters (3.4), finding that all produce identical results. We have also found that, at least in the case of ${ }^{48} \mathrm{Ca}$, there is a certain dependence of the results with respect to the spe spaces used.

In the last three rows of the upper part of the Table VII, are given the results derived for ${ }^{48} \mathrm{Ca}$ by other au- thors within the SM for the $p f$-space. ${ }^{9}$ Namely, by i) Sagawa and Uesaka [6] with GXFF1A interaction, and by ii) Auerbach and Minh Loc [7], and Shimizu, Menéndez and Yako [8], both with KB3G interaction. In fact, the values of strengths $S_{1 \mathcal{J}=0,2}^{\{+2\}}$ attributed to the last authors [8] have been extracted from their Fig. 1.

TABLE VII: Results with the $p p$ parametrization $T 1$ for: i) Fermi $(J=0 ; \mathcal{J}=0)$ and Gamow-Teller $(J=1 ; \mathcal{J}=0,2)$ DCE transition strengths $S_{J \mathcal{J}}^{\{ \pm 2\}}$ given by (2.15), ii) the corresponding sum rules $S_{J \mathcal{J}}^{\{2\}}$ calculated from(2.16), iii) the predicted sum rules $S_{J \mathcal{J}}^{\{2\}}$ given by (2.17), (2.18), and (2.19), iv) the energy centroid (2.49), and v) the transition strengths $B_{J_{J_{1}}}^{\{2\}}\left(\equiv B_{J_{\mathcal{J}_{1}}}^{\{+2\}}\right)$ for ${ }^{48} \mathrm{Ca}$ and $B_{J_{J_{1}}}^{\{2\}}\left(\equiv B_{J_{J_{1}}}^{\{-2\}}\right)$ for ${ }^{96} \mathrm{Ru}$ going to the levels $0_{1}^{+}$and $2_{1}^{+}$. The SM results from previous works [6-8] for ${ }^{48} \mathrm{Ca}$ are also shown. The meaning of the inequalities is explain in the text.

\begin{tabular}{|c|c|c|c|c|c|c|c|c|}
\hline \multicolumn{9}{|c|}{${ }^{48} \mathrm{Ca}$} \\
\hline Ref. & $J \mathcal{J}$ & $S_{J \mathcal{J}}^{\{-2\}}$ & $S_{J \mathcal{J}}^{\{+2\}}$ & $S_{J \mathcal{J}}^{\{2\}}$ & $\mathrm{S}_{J \mathcal{J}}^{\{2\}}$ & $\bar{E}_{00}^{\{-2\}}$ & $\bar{E}_{00}^{\{+2\}}$ & $\begin{array}{c}B_{J \mathcal{J}_{1}}^{\{2\}} \\
\times 10^{-3}\end{array}$ \\
\hline \multirow{3}{*}{$e_{j}^{e x p}$} & 00 & 140.1 & 0.94 & 139.1 & 112 & 20.5 & - & 77 \\
\hline & 10 & 162.2 & 2.0 & 160.2 & $\leq 175.9$ & 12.2 & 15.9 & 470 \\
\hline & 12 & 716.1 & 9.10 & 707.0 & $\geq 640.0$ & 13.2 & 16.5 & 51 \\
\hline \multirow{3}{*}{$e_{j}$} & 00 & 157.1 & 2.38 & 139.1 & 112 & 21.6 & 22.7 & 45 \\
\hline & 10 & 189.8 & 5.87 & 160.2 & $\leq 183.9$ & 14.1 & 19.7 & 163 \\
\hline & 12 & 858.5 & 26.1 & 832.4 & $\geq 752.5$ & 14.8 & 19.7 & 52 \\
\hline \multirow[t]{2}{*}[6]{} & 10 & - & - & 135.5 & $\leq 144.0$ & - & - & - \\
\hline & 12 & - & - & 501.2 & $\geq 480.0$ & - & - & - \\
\hline$[7]$ & 10 & 131.8 & - & - & $\leq 144.0$ & 21.9 & - & 0.24 \\
\hline \multirow[t]{2}{*}{ [8] } & 10 & 126.3 & - & - & - & - & - & - \\
\hline & 12 & 511.0 & - & - & - & - & - & - \\
\hline \multicolumn{9}{|c|}{${ }^{96} \mathrm{Ru}$} \\
\hline \multirow{3}{*}{$e_{j}$} & 00 & 128.0 & 0.1 & 127.9 & 112 & 21.4 & 20.4 & 0.0016 \\
\hline & 10 & 221.1 & 12.0 & 209.1 & $\leq 222.7$ & 23.1 & 13.1 & 16 \\
\hline & 12 & 981.4 & 49.4 & 932.0 & $\geq 873.4$ & 23.1 & 12.3 & 5.7 \\
\hline
\end{tabular}

Several observations are in order regarding the results shown in Table VII:

1. The strengths $S_{J \mathcal{J}}^{\{-2\}}$ are always small in comparison with the strengths $S_{J \mathcal{J}}^{\{+2\}}$ and as a consequence $S_{J \mathcal{J}}^{\{+2\}} \cong \mathrm{S}_{J \mathcal{J}}^{\{2\}}$. This is clearly due to the relatively large neutron excess.

2. Although small, the strengths $S_{J \mathcal{J}}^{\{-2\}}$ are significant in relation to the DBD. They are proportionally higher in ${ }^{96} \mathrm{Ru}$, which decays by $\beta^{+} \beta^{+}$, than in ${ }^{48} \mathrm{Ca}$, which decays by $\beta^{-} \beta^{-}$.

9 Note that the present calculations were done in a single-particle space consisting of the $2 p-1 f-2 s-1 d$ shells for both protons and neutrons. 
3. The F strengths $S_{00}^{\{2\}}$ deviate quite significantly from the sum rule strengths $\mathrm{S}_{00}^{\{2\}} ; 24 \%$ and $40 \%$, respectively, within the spe spaces $e_{j}^{e x p}$ and $e_{j}$ in ${ }^{48} \mathrm{Ca}$, and $14 \%$ in ${ }^{96} \mathrm{Ru}$. A possible explanation for these differences is given in the appendix. ${ }^{10}$

4. Terms proportional to $C$ in the GT sum rules (2.18), (2.19), and (2.20) are not included in the calculations, and this is the reason why have to be fulfilled the conditions

$$
\begin{gathered}
S_{10}^{\{2\}} \leq \mathrm{S}_{10}^{\{2\}}, \\
S_{12}^{\{2\}} \geq \mathrm{S}_{12}^{\{2\}} .
\end{gathered}
$$

In fact, they are nicely satisfied in all numerical calculations presented in Table VII.

5. All $S_{J \mathcal{J}}^{\{\mp 2\}}$ strengths depend quite significantly on the spe, but very weakly on the residual interaction. This is the reason why we only show the results for the parametrization $\mathrm{T} 1$.

6. The same situation applies to the predicted sum rules $S_{1 \mathcal{J}}^{\{2\}}$, due to their dependence on the term $S_{1}^{\{+1\}}$ (in Eqs. (2.18), (2.19) and (2.20)), which in turn depends on the spe used in the calculations.

7. The terms proportional to $C$ are omitted in (2.18), (2.19) in Refs. [6, 7], and this is the reason why their predicted sum rules $S_{1 \mathcal{J}}^{\{2\}}$ are smaller than ours.

8. Since the values for $S_{1 \mathcal{J}}^{\{+2\}}$ are not explicitly given in Shimuzu et al. [8], we have derive them from their Fig. 1b. They are consistent with the values of $S_{1 \mathcal{J}}^{\{2\}}$ presented in Refs. [6, 7].

9. Our GT strengths are always larger than those in the SM calculations. Also our average energies $\bar{E}_{1 \mathcal{J}}^{\{+2\}}$ are significantly smaller that those presented in Table II in Ref. [7], and those shown in Fig. (1b) by Shimuzu et al. [8]. It is difficult to discern whether this is due to the deficiency of our model, or the difference in the size of single-particle spaces. We are inclined to think that our results are correct, since otherwise it would be very difficult to satisfy the second condition in Eq. (3.5).

10. The size of $B_{J J_{1}}^{\{ \pm 2\}}$, shown in the last column of Table VII, and when compared with $S_{J \mathcal{J}}^{\{ \pm 2\}}$, give us an idea on the smallness of the NMEs.

${ }^{10}$ In this case it could be interesting to analyze if the problem can be solved by particle-number projection [55] .

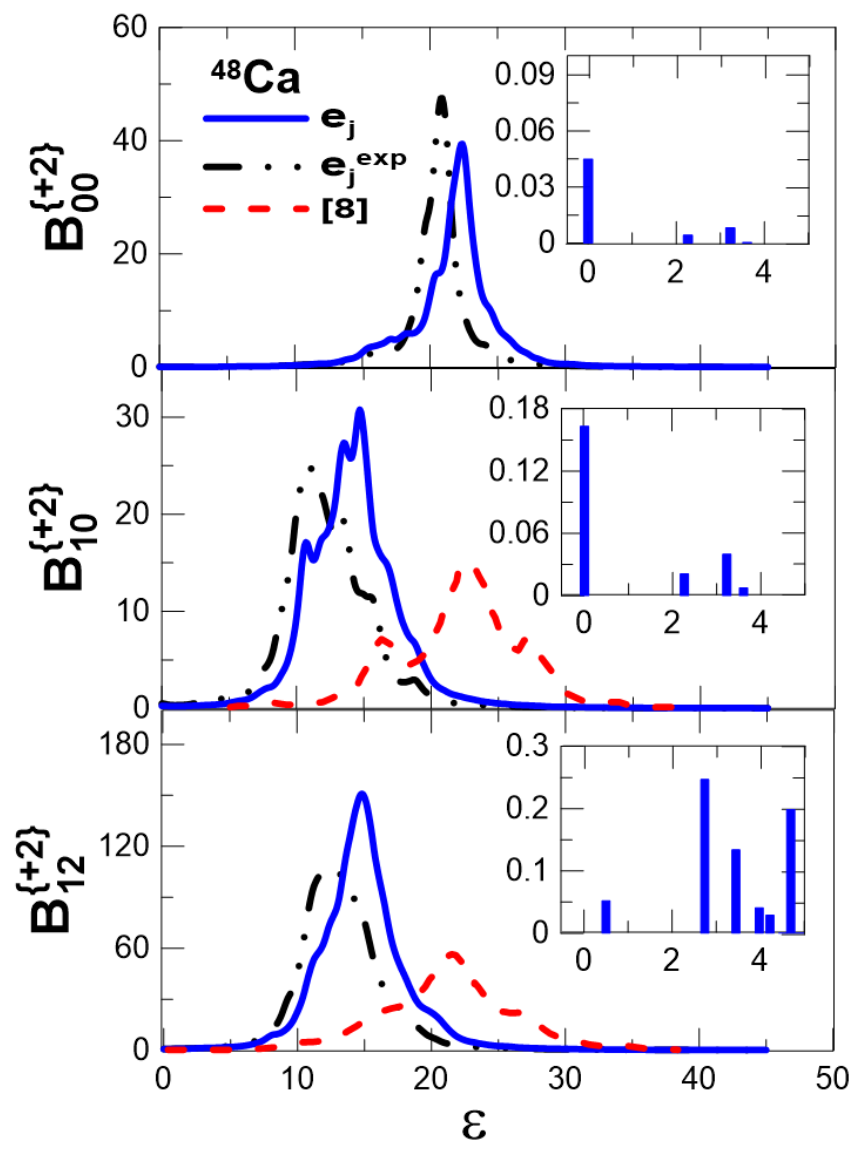

FIG. 6: DCE strength distributions $B_{J \mathcal{J}}^{\{+2\}}$ for the transition ${ }^{48} \mathrm{Ca} \rightarrow{ }^{48} \mathrm{Ti}$ with spe $e_{j}^{e x p}$, and $e_{j}^{e x p}$, and $p p$ strengths $\mathrm{T} 1$, as a function of the excitation energy $\mathcal{E}$ in ${ }^{48} \mathrm{Ti}$. The $B_{J \mathcal{J}}^{\{+2\}}$ are dimensionless, and the energies are in MeV. The SM results, obtained by Shimizu et al. [8] with the KB3G interaction, are also shown.

\section{F. Spectral Distributions of Double Charge Exchange Strengths}

The DCE strength distributions $B_{J \mathcal{J}_{f}}^{\{ \pm 2\}}$, which are of interest here, are drawn in Figs. 6-8 as a function of the excitation energy $\mathcal{E}$ in final nuclei. We have found that they depend only moderately on the spe spaces, and even less on the $p p$ parameters. To simulate the experimental energy resolution, they were smeared out with Lorentzians of $1 \mathrm{MeV}$ width. Moreover, these figures contain inserts which show the corresponding strengths in the low-lying states of final nuclei.

In the upper panel of Fig. 6 are shown the $\mathrm{F}$ distributions $B_{00}^{\{+2\}}$ in ${ }^{48} \mathrm{Ti}$, exhibiting at around $22 \mathrm{MeV}$ a fairly narrow double giant $\mathrm{F}$ resonance, usually called double isobaric analog state (DIAS). In the middle and lower panels of this figure are shown the GT distributions $B_{10}^{\{+2\}}$ and $B_{12}^{\{+2\}}$, respectively, in the same final nucleus, which also exhibit resonant like structure. These dou- 


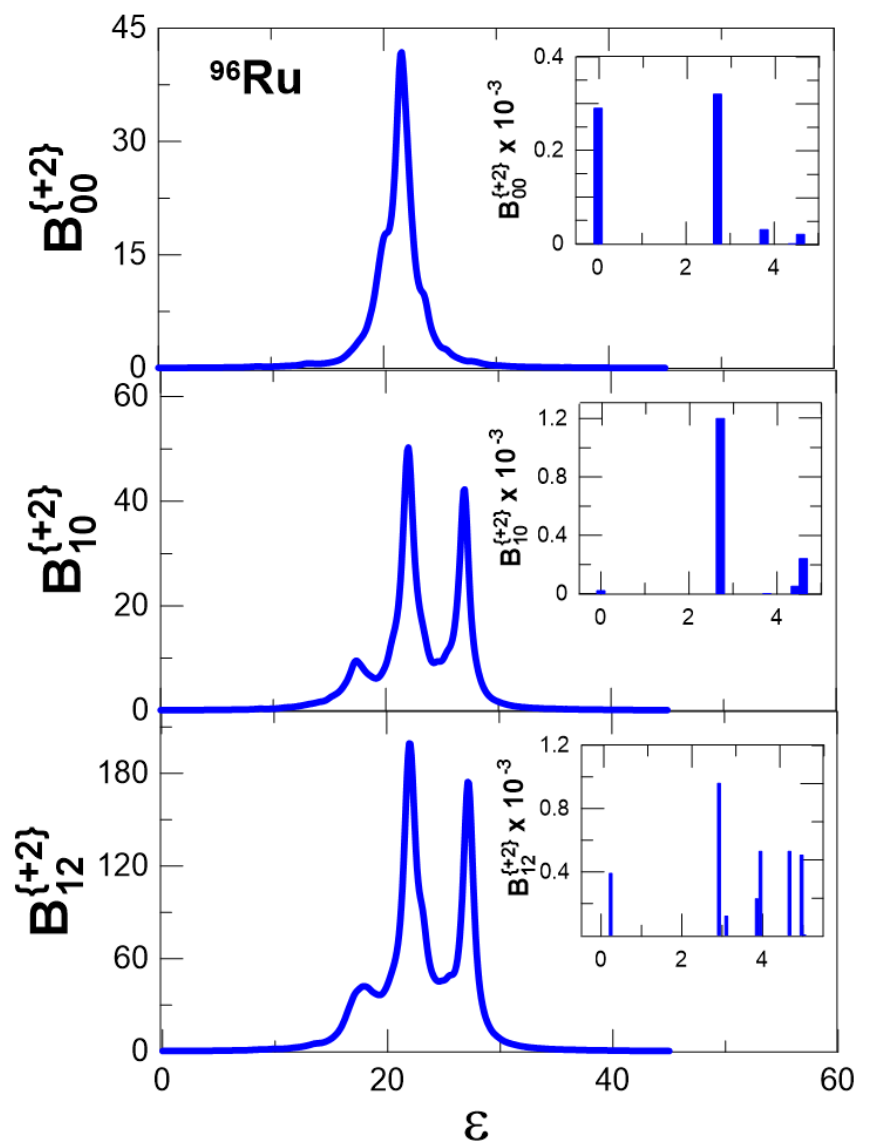

FIG. 7: DCE strength distributions $B_{J \mathcal{J}}^{\{+2\}}$ for the transition ${ }^{96} \mathrm{Ru} \rightarrow{ }^{96} \mathrm{Pd}$. The $B_{J \mathcal{J}}^{\{-2\}}$ are dimensionless, and the energies are in $\mathrm{MeV}$.

ble GT giant resonances (DGTGR) are much wider than the DIAS and centered around 13 and $14 \mathrm{MeV}$, respectively. In the KB3G SM calculations of Shimizu et al. [8], which are also shown in Fig. 6, these resonances appear at around $20 \mathrm{MeV}$.

In Figs. 7 and 8 are presented analogous results for the $B_{J \mathcal{J}}^{\{+2\}}$ and $B_{J \mathcal{J}}^{\{-2\}}$ densities in ${ }^{96} \mathrm{Pd}$ and ${ }^{96}$ Mo final nuclei, respectively. Both are shown because here we are interested in the $D B D^{+}$, where the low-energy behavior of $B_{J \mathcal{J}}^{\{-2\}}$ densities is relevant.

As seen in Fig. 7, the DIAS in ${ }^{96} \mathrm{Pd}$ is located at around $21 \mathrm{MeV}$, while both $\mathcal{J}=0^{+}$and $\mathcal{J}=2^{+}$DGTGR are at about $23 \mathrm{MeV}$. These resonances are not directly related to the $\mathrm{DBD}$ of ${ }^{96} \mathrm{Ru}$, but their locations in ${ }^{96} \mathrm{Pd}$ can be searched through heavy ion reactions.

The smallness of $B_{00}^{\{-2\}}$ and its energy distribution, shown in Fig. 8, are fully consistent with the small value of $M_{F}^{2 \nu}\left(0_{1}^{+}\right)$in Table III and of $B_{00_{1}}^{\{-2\}}$ in Table VII. Moreover, the distributions of the $B_{1 \mathcal{J}=0,2}^{\{-2\}}$ clearly indicate that the $\mathrm{DBD}^{+}$of ${ }^{96} \mathrm{Ru}$ will be very slow.

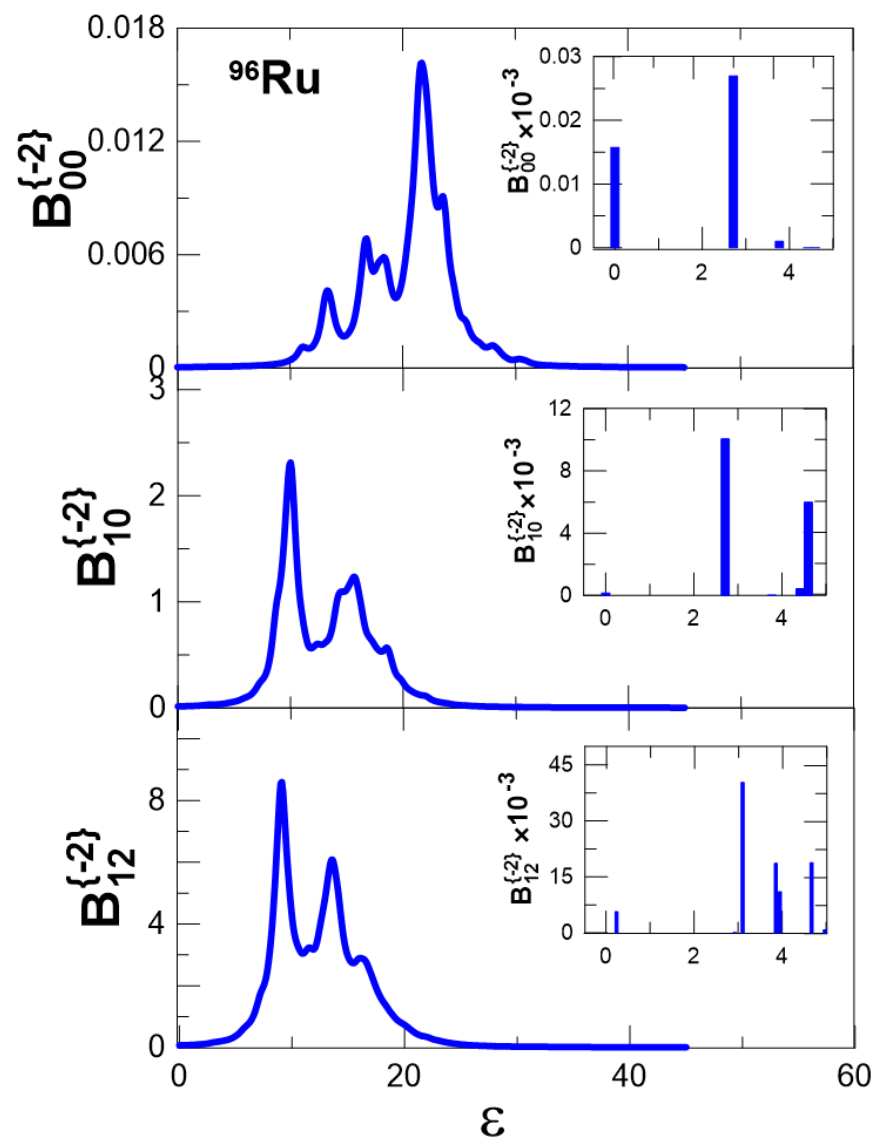

FIG. 8: DCE strength distributions $B_{J \mathcal{J}}^{\{-2\}}$ for the transition ${ }^{96} \mathrm{Ru} \rightarrow{ }^{96} \mathrm{Mo}$. The $B_{J \mathcal{J}}^{\{-2\}}$ are dimensionless, and the energies are in $\mathrm{MeV}$.

\section{G. Comparison between ground state $2 \nu$-DBD NME and DCE strengths}

In the so-called closure approximation, the sum of the intermediate states $J_{\alpha}$ in (2.1) and (2.9) are taken by closure, after replacing $E_{J_{\alpha}}^{\{\mp 1\}}$ in (2.3) by some average $\bar{E}_{J}^{\{\mp 1\}}[44]$.

Thus, except for the constant energy denominator, the ground state DCE densities $B_{J 0_{1}}^{\{ \pm 2\}}$ are the closure approximations of the squares of the NMEs. In view of this, to know how reasonable the closure approximation is, it may be interesting to compare the behaviors of these two quantities as a function of the $p p$ parameters. As an example, in Fig. 9 are compared the squares of the NMEs $M_{F}^{2 \nu}\left(0_{1}^{+}\right)$and $M_{G T}^{2 \nu}\left(0_{1}^{+}\right)$with $B_{00_{1}}^{\{+2\}}$, for ${ }^{48} \mathrm{Ca}$ with the spe $e_{j}$. The squares of the NMEs are in natural units, while the strengths are dimensionless. The proportionality between these two observables suggests that the closure approximation in the case of ${ }^{48} \mathrm{Ca}$ is reasonable. However, there is no guarantee that this result will be valid in general. 

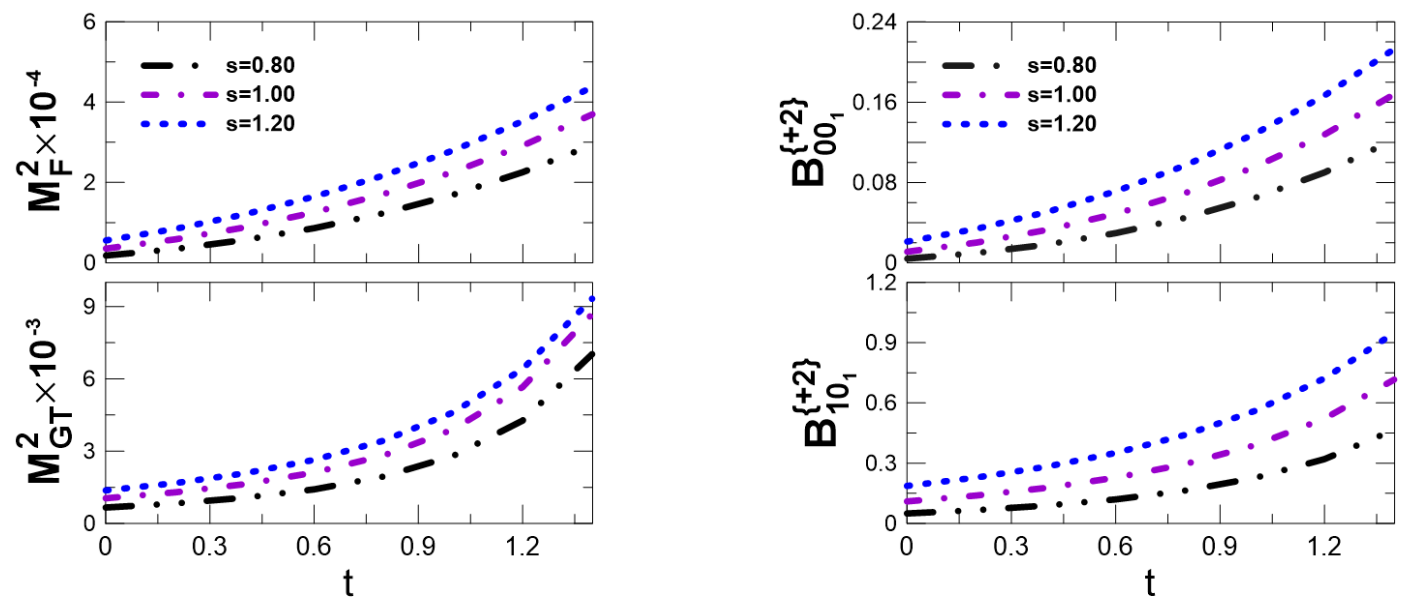

FIG. 9: (Color online) Comparison of $\left|M_{F}^{2 \nu}\left(0_{1}^{+}\right)\right|^{2}$ and $\left|M_{G T}^{2 \nu}\left(0_{1}^{+}\right)\right|^{2}$ (both in natural units) with $B_{00_{1}}^{\{+2\}}$ and $B_{10_{1}}^{\{+2\}}$ (dimensionless), respectively, in ${ }^{48} \mathrm{Ca}$ for the spe $e_{j}$.

\section{FINAL REMARKS}

We have developed a nuclear structure model that involves (pn,2p2n)-QTDA excitations on the BCS meanfield, which is capable of simultaneously describing the DBD and the DCE transition strengths. So far, this has been done only in the context of SM, where these two problems are generally treated separately, although it is well known that they are intimately related to each other. This is the case, for instance, of ${ }^{48} \mathrm{Ca}$, where the $\mathrm{DBD}^{-} \mathrm{s}$ are described in Refs. $[17,41]$, while the transition strength distributions $B_{J \mathcal{J}}^{\{+2\}}$ and the corresponding total strengths $S_{J \mathcal{J}}^{\{+2\}}$ were evaluated in Refs. [6-8] .

The (pn,2p2n)-QTDA model has additional advantages over the standard pn-QRPA model. Namely:

1. Together with the NMEs of the ground state, the NMEs of all the excited states $0^{+}$and $2^{+}$are calculated simultaneously. To do the same in the pn-QRPA model, it is necessary to resort to supplementary calculations through several chargeconserving QRPAs, thus introducing several new model parameters.

2. It allows the evaluation of the $Q$-values for DBDs, which plays a very important role in this type of processes.

The proposed model can be viewed as a natural extension to DCE processes of the pn-QRPA model, originally developed by HS to describe the SCE processes [2]. The first does not include the GSC like the second. But this is not a serious inconvenience since, as we have discussed above, the quenching mechanism is now different.

Our next aim is to evaluate and discuss the $0 \nu$ NMEs (2.12) making use of the replacement (2.11) in our previous work [19]. One expects that the relationship between DCE nuclear reactions and DBD will be more clearly visible at $0 \nu$ than at $2 \nu$ reactions, due to a lower dependence on the NME of their energy denominators in the first case.

During the development of the present study, Santopinto et al.[56], based on a previous work of Bertulani [57], have reported that, in the low-momentumtransfer limit, the heavy ion ${ }^{40} \mathrm{Ca}\left({ }^{18} \mathrm{O},{ }^{18} \mathrm{Ne}\right){ }^{40} \mathrm{Ar}$ cross section behaves as

$$
\frac{d \sigma}{d \Omega} \sim\left|\frac{\mathcal{M}_{T \rightarrow T^{\prime}}^{D G T} \mathcal{M}_{P \rightarrow P^{\prime}}^{D G T}}{\bar{E}_{P}^{G T}+\bar{E}_{T}^{G T}}+\frac{\mathcal{M}_{T \rightarrow T^{\prime}}^{D F} \mathcal{M}_{P \rightarrow P^{\prime}}^{D F}}{\bar{E}_{P}^{F}+\bar{E}_{T}^{F}}\right|^{2},
$$

where $P$ and $T$ stand for projectile and target nuclei respectively.

The correspondence with our notation is:

1) For the matrix elements 11

$$
\begin{array}{cc}
\mathcal{M}_{P \rightarrow P^{\prime}}^{D G T} \rightarrow B_{10_{1}}^{\{+2\}}, & \mathcal{M}_{P \rightarrow P^{\prime}}^{D F} \rightarrow B_{00_{1}}^{\{+2\}}, \\
\mathcal{M}_{T \rightarrow T^{\prime}}^{D G T} \rightarrow B_{10_{1}}^{\{-2\}}, & \mathcal{M}_{T \rightarrow T^{\prime}}^{D F} \rightarrow B_{00_{1}}^{\{+2\}} .
\end{array}
$$

2) For the energies (see the denominator in (2.2))

$$
\begin{aligned}
& E_{P}^{G T} \rightarrow E_{1_{\alpha}}^{\{+1\}}-E_{0^{+}}^{\{0\}}, E_{P}^{F} \rightarrow E_{0_{\alpha}}^{\{+1\}}-E_{0^{+}}^{\{0\}}, \\
& E_{T}^{G T} \rightarrow E_{1_{\alpha}}^{\{-1\}}-E_{0^{+}}^{\{0\}}, E_{T}^{F} \rightarrow E_{0_{\alpha}}^{\{-1\}}-E_{0^{+}}^{\{0\}} .
\end{aligned}
$$

Therefore, the present model posses all the necessary ingredients to evaluate the heavy-ion cross section in the low-momentum-transfer limit. Of course, now it is necessary to solve two eigenvalue problems, one for the target nucleus ${ }^{40} \mathrm{Ca}$, and one for the projectile nucleus ${ }^{18} \mathrm{O}$.

In summary, we have developed a new model, based on the BCS approach, to describe the double-charge exchange nuclear phenomena $(A, Z) \rightarrow(A, Z \pm 2)$. It is a natural extension of the Halbleib and Sorensen [2] model, aimed to describe the single-charge exchange processes

${ }^{11}$ Except for the coupling constants $c_{G T}$ and $c_{G T}$ in Eqs. (11) and (12) respectively. 
$(A, Z) \rightarrow(A, Z \pm 1)$. As an example, detailed numerical calculations are presented for the $(A, Z) \rightarrow(A, Z+2)$ process in ${ }^{48} \mathrm{Ca} \rightarrow{ }^{48} \mathrm{Ti}$ and the $(A, Z) \rightarrow(A, Z-2)$ process in ${ }^{96} \mathrm{Ru} \rightarrow{ }^{96} \mathrm{Mo}$, involving all final $0^{+}$states and $2^{+}$ states. At the moment we are extending this study in two directions:

1. A throughout evaluation of all $2 \nu$ - $\mathrm{DBD}^{ \pm}$, together with the associate nuclear reaction strengths will performed.

2. The $2 \nu$ - $\mathrm{DBD}^{ \pm}$formalism developed here will be extended to the $0 \nu-\mathrm{DBD}^{ \pm}$.

\section{Acknowledgments}

This study was financed in part by the Coordenação de Aperfeiçoamento de Pessoal de Nível Superior Brasil (CAPES) Finance Code 001. A.R.S. acknowledges the financial support of FAPESB (Fundação de Amparo à Pesquisa do Estado Bahia) TERMO DE OUTORGAPIE0013/2016. The authors thank the partial support of UESC (PROPP 00220.1300.1832). We sincerely thank to Wayne Seale for his very careful and judicious reading of the manuscript. We also thank N. Paar for providing us the spe for ${ }^{96} \mathrm{Ru}$, evaluated within the (DD-ME2) model, and to C. Bertulani and C. Barbero for stimulating comments and discussions.

\section{Appendix: A Toy Model}

In order to understand why F DCESR is not completely satisfied in our model, we resort to a toy model corresponding to the ${ }^{14} \mathrm{C}$ nucleus and considering the levels $1 s_{1 / 2}, 1 p_{1 / 2}$, and $1 p_{3 / 2}$, of which the three are totally occupied by neutrons, while only the first two are partially occupied by protons. From (2.14) we have

$$
\begin{aligned}
S_{00}^{\{-2\}} & =\sum_{f} B_{00_{f}}^{\{-2\}} \\
& =\sum_{f}\left|\sum_{\alpha}\left\langle 0_{f}^{+}\left\|\mathcal{O}_{0}^{-}\right\| 0_{\alpha}^{+}\right\rangle\left\langle 0_{\alpha}^{+} \| \mathcal{O}_{0}^{-} \mid 0_{i}^{+}\right\rangle\right|^{2} .(\mathrm{A} .1)
\end{aligned}
$$

In the BCS approximation one gets

$$
\begin{aligned}
& B_{00_{1}}^{\{-2\}}=4 u_{1 p_{1 / 2}}^{4}, B_{00_{2}}^{\{-2\}}=4 u_{1 s_{1 / 2}}^{2} u_{1 p_{1 / 2}}^{2}, \\
& B_{00_{3}}^{\{-2\}}=12 u_{1 s_{1 / 2}}^{2} u_{1 p_{1 / 2}}^{2}, B_{00_{4}}^{\{-2\}}=4 u_{1 s_{1 / 2}}^{4}
\end{aligned}
$$

and

$$
S_{00}^{\{-2\}} \equiv S_{F}^{2 \beta^{-}}=4+8 u_{1 s_{1 / 2}}^{2} u_{1 p_{1 / 2}}^{2}
$$

since $u_{1 s_{1 / 2}}^{2}+u_{1 p_{1 / 2}}^{2}=1$. For instance, with $u_{1 s_{1 / 2}}^{2}=0.95$ and $u_{1 p_{1 / 2}}^{2}=0.05$, one gets $S_{00}^{\{-2\}}=4.382$, instead of the predicted value $S_{00}^{\{-2\}}=4$. The result (A.2) also is valid when the residual interaction is switched on. This means that the F DCESR is fully satisfied only in the particlehole limit, i.e. when one of the protons $1 s_{1 / 2}, 1 p_{1 / 2}$ levels is totally full or totally empty.
[1] F. Krmpotić, Fizika B 14, 139 (2005). http://fizika.hfd.hr/fizika_b/bv05/b14p139.htm

[2] J. A. Halbleib and R. A. Sorensen, Nucl. Phys. A 98, 542 (1967). https://doi.org/10.1016/0375-9474(67)90098-X

[3] A. S. Barabash, Physics of Elementary Particles and Atomic Nucleus 42, 1183 (2011). http://www1.jinr.ru/Pepan/2011_v42/v-424/10_bar.pdf; ibid Nucl. Phys. A 935, 52 (2015). https://doi.org/10.1016/j.nuclphysa.2015.01.001.

[4] F. Cappuzzello, M. Cavallaro, C. Agodi, M. Bondi, D. Carbone, A. Cunsolo, and A. Foti. Eur. Phys. J. A 51 , 145 (2015). https://doi.org/10.1140/epja/i2015-15145-5

[5] D. Carbone et al., J. Phys. Conf. Ser. 1078 (2018) no.1, 012008. https://iopscience.iop.org/article/10.1088/1742$6596 / 1078 / 1 / 012008$

[6] H. Sagawa and T. Uesaka, Phys. Rev. C 94, 064325 (2016). https://doi.org/10.1103/PhysRevC.94.064325

[7] N. Auerbach, Bui Minh Loc, Phys. Rev. C 98, 064301 (2018). https://doi.org/10.1103/PhysRevC.98.064301

[8] N. Shimizu, J. Menéndez, and K. Yako, Phys. Rev. Lett. 120, $142502 \quad$ (2018). https://doi.org/10.1103/PhysRevLett.120.142502
[9] A. Escuderos, A. Faessler, V. Rodin, F. Simkovic, J. Phys. G 37, 125108 (2010). https://iopscience.iop.org/article/10.1088/09543899/37/12/125108

[10] J. Suhonen, Phys.Rev. C 86, 024301 (2012). https://doi.org/10.1103/PhysRevC.86.024301.

[11] D. S. Delion and J. Suhonen, Phys. Rev. C95, 034330 (2017). https://doi.org/10.1103/PhysRevC.95.034330.

[12] C. Barbero, F. Krmpotić, and A. Mariano, Phys. Lett. B 345, 192 (1995). https://doi.org/10.1016/03702693(94)01592-Z.

[13] M. Hirsch, K. Muto, T. Oda, H.V. KlapdorKleingrothaus, Z. Physik A - Hadrons and Nuclei 347, 151 (1994). https://doi.org/10.1007/BF01292371

[14] J. P. Pirinen and J. Suhonen, Phys. Rev. C 91, 054309 (2015). https://doi.org/10.1103/PhysRevC.91.054309.

[15] H. Li, Z. Ren, Phys. Rev. C 96, 065503 (2017). https://doi.org/10.1103/PhysRevC.96.065503.

[16] M. Doi and T. Kotani, Prog. Theor. Phys. 87, No. 5, 1207 (1992). https://doi.org/10.1143/ptp/87.5.1207

[17] A. A. Raduta, C. M. Raduta, $\begin{array}{llll}\text { Phys.Lett. } & \text { B } & \mathbf{6 4 7}, & \end{array}$ 
http://dx.doi.org/10.1016/j.physletb.2007.02.007

[18] A. Shukla, R. Sahu, and V. K. B. Kota Phys. Rev. C 80, $057305 \quad$ (2009). https://doi.org/10.1103/PhysRevC.80.057305

[19] V. dos S. Ferreira, F. Krmpotić, C. A. Barbero, and A. R. Samana, Phys. Rev. C 96, 044322 (2017). https://doi.org/10.1103/PhysRevC.96.044322

[20] P. Vogel, M. Ericson, and J. D. Vergados, Phys. Lett. B212, 259 (1988). https://doi.org/10.1016/03702693(88)91313-5

[21] K. Muto, Phys. Lett. B 277, 13 (1992). https://doi.org/10.1016/0370-2693(92)90948-4

[22] D. C. Zheng, L. Zamick, and N. Auerbach, Phys. Rev. C 40, 936 (1989). https://doi.org/10.1103/PhysRevC.40.936.

[23] J. Suhonen, From Nucleons to Nucleus: Concepts of Microscopic Nuclear Theory (Springer, Berlin, 2007).

[24] M. K. Pal, Y. K. Gambhir, and Ram Raj, Phys. Rev. 155, 1144 (1966). https://doi.org/10.1103/PhysRev.155.1144

[25] Ram Raj and M. L. Rustgi, Phys. Rev. 178, 1556 (1969). https://doi.org/10.1103/PhysRev.178.1556.

[26] M. Baranger, Phys. Rev. 120, 957 (1960). https://doi.org/10.1103/PhysRev.120.957.

[27] A. Bohr and B.R. Mottelson, Nuclear Structure, Vol. 1 (W. A. Benjamin, New York, Amsterdam, 78, 1969).

[28] J. Kotila and F. Iachello, Phys. Rev. C 87, 024313 (2013). https://doi.org/10.1103/PhysRevC.87.024313.

[29] S. Stoica, M. Mirea, Front. Phys. 7, 12 (2019). https://doi.org/10.3389/fphy.2019.00012

[30] S. Stoica, Chinese Phys. C 43, No. 6, 064108 (2019). https://doi.org/10.1088/1674-1137/43/6/064108.

[31] K. Nakayama, A. Pio Galeão and F. Krmpotić, Phys. Lett. B 114, 217 (1982). https://doi.org/10.1016/03702693(82)90480-4.

[32] G. E. Brown and Mannque Rho, Nucl. Phys. A 372, 397 (1981). https://doi.org/10.1016/0375-9474(81)90043-9; Aage Bohr and Ben R. Mottelson, Phys. Lett. B 100, 10 (1981). https://doi.org/10.1016/0370-2693(81)902744; H. Castillo and F. Krmpotić, Nucl. Phys. A 469, 637 (1987). https://doi.org/10.1016/0375-9474(87)90018-2.

[33] S. Yoshida, Y. Utsuno, N. Shimizu, T. Otsuka, Phys.Rev. C 97, 054321 (2018). https://doi.org/10.1103/PhysRevC.97.054321.

[34] J. Beringer et al. (Particle Data Group), Phys. Rev. D $86,010001 \quad$ (2012). https://doi.org/10.1103/PhysRevD.86.010001.

[35] P. Gysbers et al., Nature Physics 15, 428 (2019). https://dx.doi.org/10.1038/s41567-019-0450-7

[36] J.T. Suhonen, Front. Phys. 5, 55 (2017), https://doi.org/10.3389/fphy.2017.00055; J. Suhonen, IOP Conf. Series: Journal of Physics: Conf. Series 1056, 012056 (2018). https://iopscience.iop.org/article/10.1088/1742$6596 / 1056 / 1 / 012056$
[37] C. Conci, V. Klemt and J. Speth, Phys. Lett. B 148, 405 (1984). https://doi.org/10.1016/0370-2693(84)90727-5

[38] N. Schwierz, I. Wiedenhover, A. Volya, arXiv:0709.3525 [nucl-th]. https://arxiv.org/abs/0709.3525v1.

[39] M. Kortelainen et al., Phys.Rev. C 89 , 054314 (2014). https://doi.org/10.1103/PhysRevC.89.054314.

[40] F.Krmpotić, A. Samana, and A.Mariano, $\begin{array}{llll}\text { Phys.Rev. } & \text { C } & \mathbf{7 1} & 044319\end{array}$ https://doi.org/10.1103/PhysRevC.71.044319.

[41] M. Horoi, S. Stoica, and B. A. Brown, $\begin{array}{lllll}\text { Phys. Rev. C } & \text { 75, } & 034303 & \text { (2007). }\end{array}$ https://doi.org/10.1103/PhysRevC.75.034303.

[42] M. Horoi, Phys. Rev. C 87, 014320 (2013). https://doi.org/10.1103/PhysRevC.87.014320.

[43] J. Kotila and F. Iachello, Phys. Rev. C 85, 034316 (2012). https://doi.org/10.1103/PhysRevC.85.034316.

[44] M.Doi, T. Kotani, H. Nishiura and E. Takasugi, Prog. Theor. Phys. 69, 602 (1983). https://doi.org/10.1143/PTP.69.602.

[45] R. Almar, O. Civitarese, and F. Krmpotić, Phys. Rev. C8, 1518 (1973). https://doi.org/10.1103/PhysRevC.8.1518.

[46] V. Paar, Nucl. Phys. A 211, 29 (1973). https://doi.org/10.1016/0375-9474(73)90763-X.

[47] N. Paar, private communication.

[48] N. Paar, D. Vretenar, T. Marketin, and P. Ring, Phys. Rev. C77, 024608 (2008). https://doi.org/10.1103/PhysRevC.77.024608

[49] A. R. Samana, F. Krmpotić, and C.A. Bertulani, Comput. Phys. Commun. 181, 1123 (2010). https://doi.org/10.1016/j.cpc.2010.02.003.

[50] M. Hirsch, M. Muto, T. Oda, H.V. KlapdorKleingrothaus, Z. Phys. A 347, 151 (1994). https://doi.org/10.1007/BF01292371.

[51] O. A. Rumyantsev and M. H. Urin, Phys. Lett. B 443, 51 (1998). https://doi.org/10.1016/S03702693(98)01291-X.

[52] P. K. Raina, A. Shukla, S. Singh, P. K. Rath, and J. G. Hirsch, Eur. Phys. J. A 28, 27 (2006). https://doi.org/10.1140/epja/i2005-10280-2.

[53] J. Barea, J. Kotila, and F. Iachello $\begin{array}{llll}\text { Phys.Rev. } & \text { C 91, } & 034304 & \text { (2015). }\end{array}$ https://doi.org/10.1103/PhysRevC.91.034304

[54] P. Belli et al., Phys. Rev. C 87, 034607 (2013). https://doi.org/10.1103/PhysRevC.87.034607

[55] F. Krmpotić, A. Mariano, T. T. S. Kuo and K. Nakayama, Phys. Lett. B 319, 393 (1993). https://doi.org/10.1016/0370-2693(93)91740-E.

[56] E. Santopinto, H. García-Tecocoatzi, R.I. Magaña Vsevolodovna, and J. Ferretti (NUMEN Collaboration), Phys.Rev. C 98, 061601 (2018). https://doi.org/10.1103/PhysRevC.98.061601.

[57] C. A. Bertulani, Nucl. Phys. A 554, 493 (1993). https://doi.org/10.1016/0375-9474(93)90232-M 